\title{
An analytical closed-form solution for free vibration of functionally graded circular plates with even and uneven porosity distributions
}

\author{
Krzysztof Kamil Żur \\ Faculty of Engineering Management \\ Bialystok University of Technology \\ Ojca Stefana Tarasiuka 2, 16-001 Kleosin \\ e-mail:k.zur@pb.edu.pl
}

\begin{abstract}
Free axisymmetric and non-axisymmetric vibration analysis of the unsaturated porous functionally graded circular plates has been presented on the basis of classical plate theory. The defined coupled equations of motion for the porous functionally graded circular plate were decoupled based on the properties of the physical neutral surface. The one general solution of the decoupled equation of motion was obtained as linear combinations of the multiparametric special Bessel functions for the functionally graded circular plate with even and uneven porosity distributions. The influences of the even and uneven distributions of porosity, gradient index, diverse boundary conditions and the negligible effect of the coupling in-plane and transverse displacements on the dimensionless frequencies of the circular plate were comprehensively studied. The obtained numerical results show the differences and significant effect of considered types of distributions of porosities, values of the gradient index and the porosity volume fraction on the distribution of eigenfrequencies of the circular plates. Additionally, the obtained multiparametric general solution of the defined differential equation will allow to study the influences of diverse additional complicating effects such as stepped thickness, cracks, additional mounted elements expressed by only additional boundary conditions on the dynamic behavior of the porous functionally graded circular/annular plates. The formulated boundary value problem, the method of solution and the obtained numerical results for the perfect and imperfect functionally graded circular plates have not yet been reported. The present paper fills this void in the literature.
\end{abstract}

Keywords: Porous FGM circular plate, Free vibration, Closed-form solution, Multiparametric characteristic equations.

\section{Introduction}

Functionally graded materials (FGMs) are a class of composite materials, which are made of the ceramic and metal mixture such that the material properties vary continuously in appropriate directions of structural components. In the processes of preparing functionally graded material, micro-voids and porosities may appear inside material in view of the technical issues. Zhu et al. [1] reported that many porosities appear in material during the functionally graded material preparation process by the nonpressure sintering technique. Wattanasakulpong et al. [2] reported that exist many porosities in the intermediate area of the functionally graded material fabricated by utilizing multi-step sequential infiltration technique because of the problem with infiltration of the secondary material into the middle area. In that case less porosities appear in the top and bottom area of material because infiltration of the material is easier in these zones.

In recent years, a significant number of articles about the free vibrations of functionally graded (FGM) plates have appeared in the literature due to their wide applications in many fields of engineering such us civil, mechanical, automotive, aeronautical and ocean engineering. The gradation of properties in functionally graded materials and the diverse distributions of porosity have a significant effect on 
distributions of the mass and the stiffness of plates and therefore their natural frequencies. The knowledge about influence of distribution of the material properties on dynamics of plates is very important because allows us to predict increasing (decreasing) the frequency of plates and find their optimal parameters. Additionally, the comprehensive investigation of the effect of functionally graded material with porosities and diverse boundary conditions on the natural frequencies of plates is the first important step to design of their safe and rational active vibration control system.

We note that in most engineering applications, the classical plate theory is often used to analysis of the dynamic behavior of thin lightweight plates. It is impossible to review all works focused on mechanical behavior of porous, FGM and porous FGM structures, then we limit to chronological reviewing some of the works focused on mechanical behavior of porous and porous FGM plates that are closely related to our work.

Jabbari et al. [3] studied the buckling of thin saturated porous circular plate with the layers of piezoelectric actuators. Buckling load was obtained for clamped circular plate under uniform radial compressive loading. The same authors presented the buckling analysis of clamped thin saturated porous circular plate with sensor-actuator layers under uniform radial compression [4]. Mojahedin et al. [5] investigated thermal and mechanical stability of clamped thin saturated and unsaturated porous circular plate with piezoelectric actuators. Rad and Shariyat [6] solved to three-dimensional magneto-elastic problem for asymmetric variable thickness porous FGM circular supported on the Kerr elastic foundation using the differential quadrature method and the state space vector technique. Barati et al. [7] studied buckling of functionally graded piezoelectric rectangular plates with porosities based on the four-variable plate theory. Mechab et al. [8] studied free vibration of FGM nanoplate with porosities resting on Winkler and Pasternak elastic foundation based on the two-variable plate theory. Mojahedin et al. [9] analyzed buckling of radially loaded clamped saturated porous circular plates based on higher order shear deformation theory. Wang and $\mathrm{Zu}$ [10] analyzed vibration behaviors of thin FGM rectangular plates with porosities and moving in thermal environment using the method of harmonic balance and the Runge-Kutta technique. Gupta and Talha [11] analyzed flexural and vibration response of porous FGM rectangular plates using nonpolynomial higher-order shear and normal deformation theory. Wang and $\mathrm{Zu} \mathrm{[12]} \mathrm{analyzed} \mathrm{vibration} \mathrm{characteristics} \mathrm{of} \mathrm{longitudinally} \mathrm{moving} \mathrm{sigmoid} \mathrm{porous}$ FGM plates based on the von Kármán non-linear plate theory. Ebrahimi et al. [13] studied free vibration of smart shear deformable rectangular plates made of porous magneto-electro-elastic functionally graded materials. Feyzi and Khorshidvand [14] studied axisymmetric pos-buckling behavior of a saturated porous circular plate with simply supported and clamped boundary conditions. Wang and $\mathrm{Zu}$ [15] studied large-amplitude vibration of thin sigmoid functionally graded plates with porosities. Wang et al. [16] studied vibrations of longitudinally travelling FGM porous thin rectangular plates using the Galerkin method and the four-order Runge-Kutta method. Ebrahimi et al. [17] used a four-variable shear deformation refined plate theory for free vibration analysis of embedded smart rectangular plates made of magneto-electro-elastic porous functionally graded materials. Shahverdi and Barati [18] developed nonlocal strain-gradient elasticity model for vibration analysis of porous FGM nano-scale rectangular plates. Shojaeefard et al. [19] studied free vibration and thermal buckling of micro temperaturedependent FGM porous circular plate using the generalized differential quadrature method. Barati and Shahverdi [20] presented new solution to examine large amplitude vibration of a porous nanoplate resting on a nonlinear elastic foundation modeled by nonlinear the four-variable plate theory. Kiran et al. [21] studied free vibration of porous FGM magneto-electro-elastic skew plates using the finite element formulation. Cong et al. [22] presented analytical approach to buckling and post-buckling behavior analysis of FGM rectangular plates with porosities under thermal and thermomechanical loads based on the Reddy's higher-order shear deformation theory. Kiran and Kattimani [23] studied free vibration and static behavior of porous FGM magneto-electro-elastic rectangular plates using the finite element method. Arshid and Khorshidvand [24] analyzed free vibration of saturated porous FGM 
circular plates integrated with piezoelectric actuators using the differential quadrature method. Shahsavari et al. [25] used the quasi-3D hyperbolic theory for free vibration of porous FGM rectangular plates resting on Winkler, Pasternak and Kerr foundations.

\section{Present study}

The aim of the paper is to formulate and solve the boundary value problem for the free axisymmetric and non-axisymmetric vibrations of FGM circular plates with even and uneven porosity distributions and diverse boundary conditions. The defined coupled equations of motion for the porous FGM circular plate were decoupled based on the properties of physical neutral surface [26]. The general solution of the obtained decoupled equation of motion of porous FGM circular plate was defined as the linear combination of the Bessel functions dependent on the material parameters. The derived multiparametric non-linear characteristic equations have universal forms because they are functionally dependent on the power-law index, the porosity volume fraction, the Young's modulus and the densities of porous functionally graded material. The presented characteristic equations allow us to comprehensively study the effect of the distribution of material parameters and the formulated boundary conditions on the natural frequencies of axisymmetric and non-axisymmetric vibrations of the circular plates without the necessity to solve a new eigenvalue problem for plates with steady distribution of parameters.

Authors of many previous papers (e.g. [27], [28], [29], [30]) presented the free vibration analysis of the perfect (without porosity) FGM circular plates using the equation of motion including only the coefficient of pure bending stiffness varying in the thickness direction of the plate, but the coefficients of extensional stiffness and bending-extensional coupling stiffness were neglected because the effect of the coupled in-plane and transverse displacements was omitted. As a result, the authors obtained the approximate model of functionally graded material and considered its effect on the natural frequencies of circular plates with the additional complicating effects.

In the present paper the obtained equation of motion of the perfect and imperfect FGM circular plates includes the coefficients of extensional stiffness, bending-extensional coupling stiffness and bending stiffness, which appeared by decoupling the in-plane and transverse displacements [31] using the properties of the physical neutral surface. The differences between the values of numerical results for the eigenfrequencies of the perfect FGM circular plate with and without the coupling effect are shown for diverse boundary conditions.

A review of literature shows that there are no studies which focus on the free axisymmetric and non-axisymmetric vibrations of FGM and porous FGM circular plates based on the classical plate theory and the properties of physical neutral surface. In particular, the obtained exact solution, the multiparametric characteristic equations and the calculated eigenfrequencies for the free vibrations of perfect and imperfect FGM circular plates with clamped, simply supported, sliding and free edges have not yet been reported. The present paper fills this void in the literature.

\section{FGM circular plate with porosities}

Consider a porous FGM thin circular plate with radius $R$ and thickness $h$ presented in the cylindrical coordinate $(r, \theta, z)$ with the z-axis along the longitudinal direction. The geometry and the coordinate system of the considered circular plate are shown in Fig. 1. The FGM plate contains evenly $(e)$ and unevenly $(u)$ distributed porosities along the plate's thickness direction. The cross-sections of the FGM circular plates with the two various types of distribution of porosities are shown in Fig. 2. 


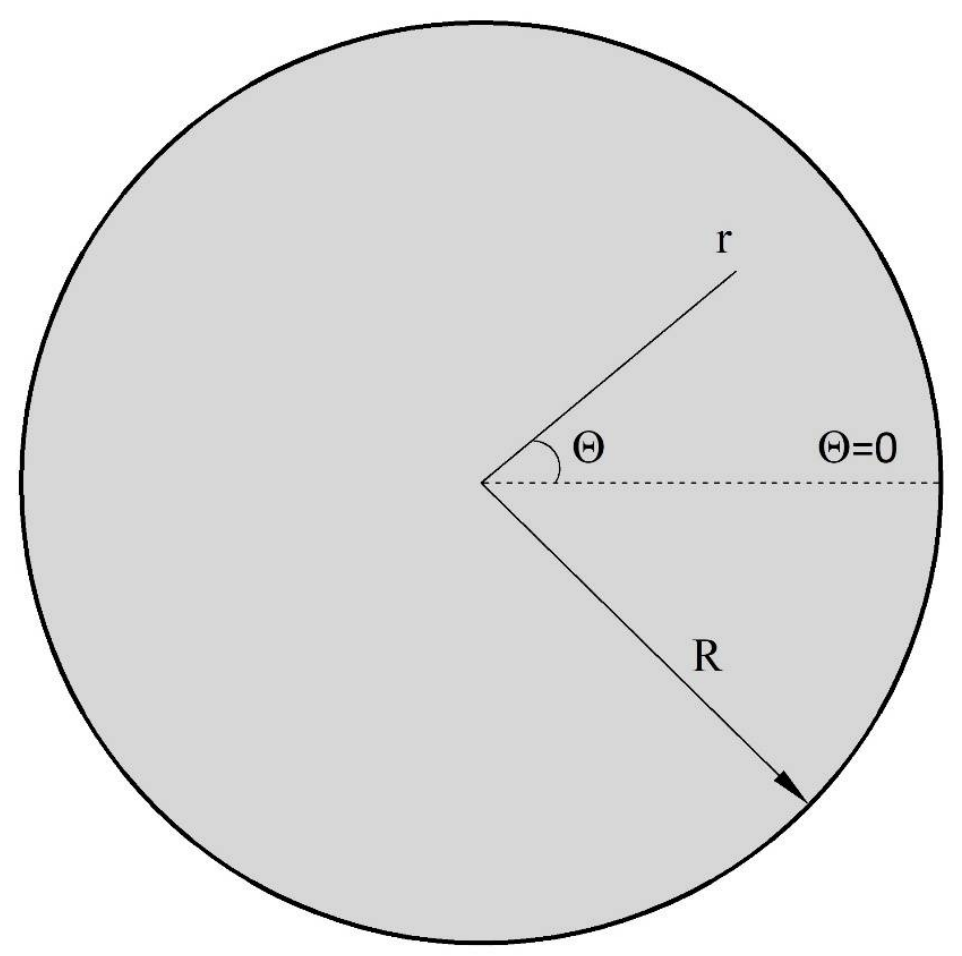

Fig. 1. The geometry and the coordinate system of the porous FGM circular plate.

a)

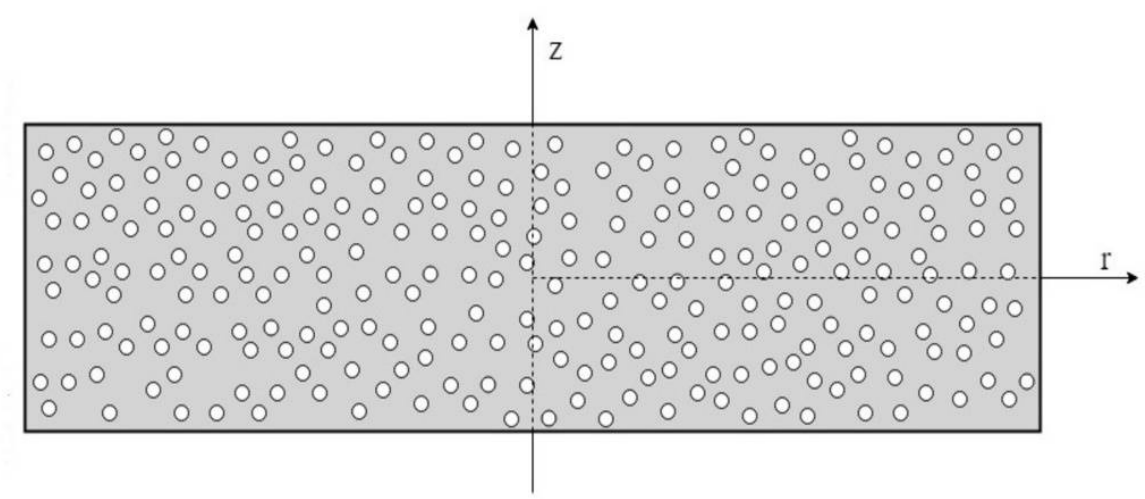

b)

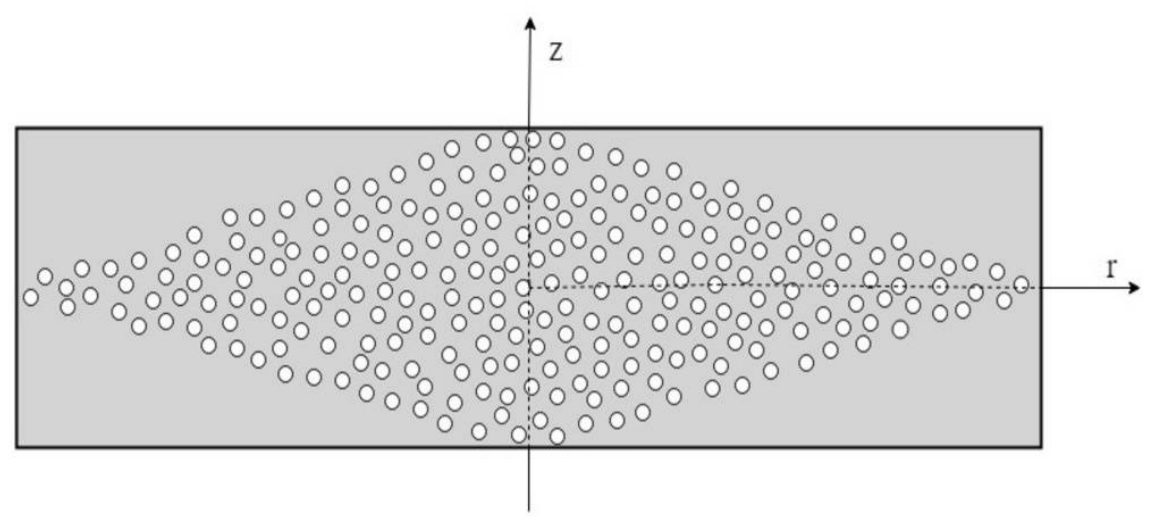

Fig. 2. The cross-sections of the porous FGM circular plate: a) even distribution; b) uneven distribution. 
The functionally graded material is a mixture of a ceramic $(c)$ and a metal $(m)$. If the volume fraction of the ceramic part is $V_{c}$ and the metallic part is $V_{m}$, we have the well-known dependence:

$V_{c}(z)+V_{m}(z)=1$.

Based on the modified rule of mixtures [16] with the porosity volume fraction $\psi(\psi \ll 1)$, the Young's modulus, the density and the Poisson's ratio for evenly $(e)$ distributed porosities over the cross-section of the plate have the general forms:

$E^{e}(z, \psi)=E_{c}\left[V_{c}(z)-\frac{\psi}{2}\right]+E_{m}\left[V_{m}(z)-\frac{\psi}{2}\right]$,

$\rho^{e}(z, \psi)=\rho_{c}\left[V_{c}(z)-\frac{\psi}{2}\right]+\rho_{m}\left[V_{m}(z)-\frac{\psi}{2}\right]$,

$v^{e}(z, \psi)=v_{c}\left[V_{c}(z)-\frac{\psi}{2}\right]+v_{m}\left[V_{m}(z)-\frac{\psi}{2}\right]$.

The volume fraction of the ceramic part changes continually along the thickness and can be defined as [32]

$V_{c}(z, g)=\left(\frac{z}{h}+\frac{1}{2}\right)^{g}, g \geq 0$,

where $g$ is the power-law index of the material. A change in the power $g$ of functionally graded material results in a change in the portion of the ceramic and metal components in the circular plate. We assume that the composition is varied from the bottom surface $(z=-h / 2)$ to the top surface $(z=h / 2)$ of the circular plate. After substituting the variation of the ceramic part $V_{c}(z, g)$ from Eq. (3) into Eqs. (2), the material properties of the functionally graded circular plate with evenly distributed porosities are defined in the final form:

$E^{e}(z, g, \psi)=\left(E_{c}-E_{m}\right)\left(\frac{z}{h}+\frac{1}{2}\right)^{g}+E_{m}-\frac{\psi}{2}\left(E_{c}+E_{m}\right)$

$\rho^{e}(z, g, \psi)=\left(\rho_{c}-\rho_{m}\right)\left(\frac{z}{h}+\frac{1}{2}\right)^{g}+\rho_{m}-\frac{\psi}{2}\left(\rho_{c}+\rho_{m}\right)$,

$v^{e}(z, g, \psi)=\left(v_{c}-v_{m}\right)\left(\frac{z}{h}+\frac{1}{2}\right)^{g}+v_{m}-\frac{\psi}{2}\left(v_{c}+v_{m}\right)$.

For the functionally graded circular plate with unevenly $(u)$ distributed porosities [16], the material properties in Eqs. (4) can be replaced by the following forms:

$E^{u}(z, g, \psi)=\left(E_{c}-E_{m}\right)\left(\frac{z}{h}+\frac{1}{2}\right)^{g}+E_{m}-\frac{\psi}{2}\left(E_{c}+E_{m}\right)\left(1-\frac{2|z|}{h}\right)$,

$\rho^{u}(z, g, \psi)=\left(\rho_{c}-\rho_{m}\right)\left(\frac{z}{h}+\frac{1}{2}\right)^{g}+\rho_{m}-\frac{\psi}{2}\left(\rho_{c}+\rho_{m}\right)\left(1-\frac{2|z|}{h}\right)$,

$v^{u}(z, g, \psi)=\left(v_{c}-v_{m}\right)\left(\frac{z}{h}+\frac{1}{2}\right)^{g}+v_{m}-\frac{\psi}{2}\left(v_{c}+v_{m}\right)\left(1-\frac{2|z|}{h}\right)$.

In this case the porosity linearly decrease to zero at the top and the bottom of the cross-section of the plate. The effect of Poisson's ratio is much less on the mechanical behavior of FGM plates [33, 34] than the Young's modulus, thus the Poisson's ratio will assume to be constant $v^{e}=v^{u}=v$ in the whole volume of the porous FGM circular plate.

\section{Constitutive relations and governing equations}

In most practical applications, the ratio of the radius $R$ to the thickness $h$ of the plate is more than 10 , then the assumptions of classical plate theory (CPT) are applicable and rotary inertia and shear deformation can be successfully omitted.

For a thin circular plate, the displacement field has the form:

$u_{r}(r, \theta, z, t)=u(r, \theta, t)-z \frac{\partial w(r, \theta, t)}{\partial r}$, 
$u_{\theta}(r, \theta, z, t)=v(r, \theta, t)-\frac{z}{r} \frac{\partial w(r, \theta, t)}{\partial \theta}$

$w(r, \theta, z, t)=w(r, \theta, t)$,

where $u, v$ and $w$ are the radial, circumferential and transverse displacements of the midplane $(z=0)$ of the plate at time $t$. Based on the linear strain-displacement relations and Hook's law, the resultant forces and the moments for porous FGM circular plate $(i=\{e, u\})$ can be expressed in the following form [35]:

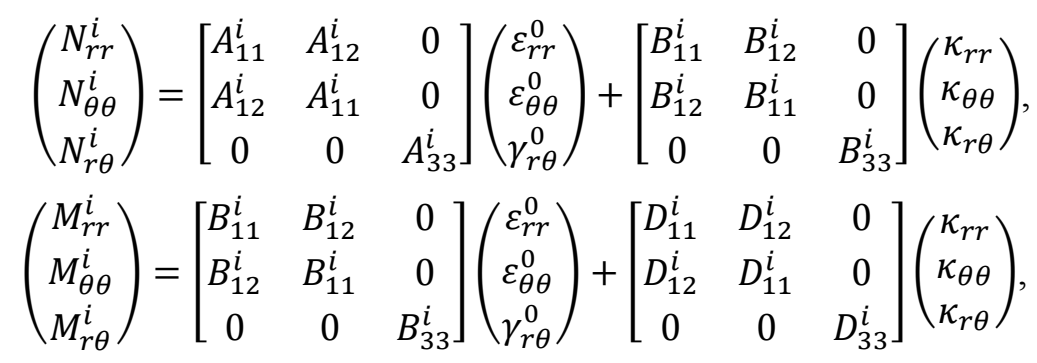

where

$$
\begin{aligned}
& \left(\varepsilon_{r r}^{0}, \varepsilon_{\theta \theta}^{0}, \gamma_{r \theta}^{0}\right)=\left(\frac{\partial u}{\partial r}, \frac{1}{r} \frac{\partial v}{\partial \theta}+\frac{u}{r}, \frac{1}{r} \frac{\partial u}{\partial \theta}+\frac{\partial v}{\partial r}-\frac{v}{r}\right), \\
& \left(\kappa_{r r}, \kappa_{\theta \theta}, \kappa_{r \theta}\right)=\left(-\frac{\partial^{2} w}{\partial r^{2}},-\frac{1}{r^{2}} \frac{\partial^{2} w}{\partial \theta^{2}}-\frac{1}{r} \frac{\partial w}{\partial r},-\frac{2}{r} \frac{\partial^{2} w}{\partial r \partial \theta}+\frac{2}{r^{2}} \frac{\partial w}{\partial \theta}\right)
\end{aligned}
$$

are the in-plane strains and curvatures of midplane, respectively.

We assume that the material properties are varied from the bottom surface $(z=-h / 2)$ to the top surface $(z=h / 2)$ of the plate, then the coefficients of extensional stiffness $A_{k l}^{i}$, bending-extensional coupling stiffness $B_{k l}^{i}$ and bending stiffness $D_{k l}^{i}$ can be defined for FGM circular plate with $i$-th distribution of porosities in the general forms:

$$
\begin{aligned}
& \left(A_{11}^{i}, B_{11}^{i}, D_{11}^{i}\right)=\int_{-h / 2}^{h / 2} \frac{E^{i}(z, g, \psi)}{1-v^{2}}\left(1, z, z^{2}\right) d z, \\
& \left(A_{12}^{i}, B_{12}^{i}, D_{12}^{i}\right)=\int_{-h / 2}^{h / 2} \frac{v E^{i}(z, g, \psi)}{1-v^{2}}\left(1, z, z^{2}\right) d z, \\
& \left(A_{33}^{i}, B_{33}^{i}, D_{33}^{i}\right)=\int_{-h / 2}^{h / 2} \frac{E^{i}(z, g, \psi)}{2(1+v)}\left(1, z, z^{2}\right) d z .
\end{aligned}
$$

Additionally, the stiffness coefficients from Eqs. (9) satisfy the equations

$A_{12}^{i}+2 A_{33}^{i}=A_{11}^{i}, B_{12}^{i}+2 B_{33}^{i}=B_{11}^{i}, D_{12}^{i}+2 D_{33}^{i}=D_{11}^{i}$.

The resultant forces and the moments can be also defined by

$$
\begin{aligned}
& \left(N_{r r}^{i}, N_{\theta \theta}^{i}, N_{r \theta}^{i}\right)=\int_{-h / 2}^{h / 2}\left(\sigma_{r r}^{i}, \sigma_{\theta \theta}^{i}, \tau_{r \theta}^{i}\right) d z, \\
& \left(M_{r r}^{i}, M_{\theta \theta}^{i}, M_{r \theta}^{i}\right)=\int_{-h / 2}^{h / 2}\left(\sigma_{r r}^{i} Z, \sigma_{\theta \theta}^{i} Z, \tau_{r \theta}^{i} Z\right) d z,
\end{aligned}
$$

where the stress components and the strain components have the form:

$$
\begin{aligned}
& \left(\begin{array}{c}
\sigma_{r r}^{i} \\
\sigma_{\theta \theta}^{i} \\
\tau_{r \theta}^{i}
\end{array}\right)=\left(\begin{array}{c}
\frac{E^{i}(z, g, \psi)}{1-v^{2}}\left(\varepsilon_{r r}+v \varepsilon_{\theta \theta}\right) \\
\frac{E^{i}(z, g, \psi)}{1-v^{2}}\left(\varepsilon_{\theta \theta}+v \varepsilon_{r r}\right) \\
\frac{E^{i}(z, g, \psi)}{2(1+v)}\left(2 \gamma_{r \theta}\right)
\end{array}\right), \\
& \left(\begin{array}{c}
\varepsilon_{r r} \\
\varepsilon_{\theta \theta} \\
2 \gamma_{r \theta}
\end{array}\right)=\left(\begin{array}{c}
\varepsilon_{r r}^{0}+z \kappa_{r r} \\
\varepsilon_{\theta \theta}^{0}+z \kappa_{\theta \theta} \\
\gamma_{r \theta}^{0}+z \kappa_{r \theta}
\end{array}\right) .
\end{aligned}
$$




\subsection{Coupled equations of motion}

Using the Hamilton's principle [35] and ignoring in-plane inertia forces, the equilibrium equations of motion of the porous FGM thin circular plate have the forms:

$\frac{\partial N_{r r}^{i}}{\partial r}+\frac{1}{r}\left(\frac{\partial N_{r \theta}^{i}}{\partial \theta}+N_{r r}^{i}-N_{\theta \theta}^{i}\right)=0$,

$\frac{\partial N_{r \theta}^{i}}{\partial r}+\frac{1}{r} \frac{\partial N_{\theta \theta}^{i}}{\partial \theta}+\frac{2}{r} N_{r \theta}^{i}=0$,

$\frac{\partial^{2} M_{r r}^{i}}{\partial r^{2}}+\frac{2}{r} \frac{\partial M_{r r}^{i}}{\partial r}+\frac{1}{r^{2}} \frac{\partial^{2} M_{\theta \theta}^{i}}{\partial \theta^{2}}-\frac{1}{r} \frac{\partial M_{\theta \theta}^{i}}{\partial r}+\frac{2}{r} \frac{\partial^{2} M_{r \theta}^{i}}{\partial r \partial \theta}+\frac{2}{r^{2}} \frac{\partial M_{r \theta}^{i}}{\partial \theta}=\rho^{i} h \frac{\partial^{2} w}{\partial t^{2}}$,

where the resultants forces and the moments can be obtain using Eqs. (7) and (8), and can be presented in the following form:

$N_{r r}^{i}=A_{11}^{i} \frac{\partial u}{\partial r}+A_{12}^{i}\left(\frac{1}{r} \frac{\partial v}{\partial \theta}+\frac{u}{r}\right)-B_{11}^{i} \frac{\partial^{2} w}{\partial r^{2}}-B_{12}^{i}\left(\frac{1}{r^{2}} \frac{\partial^{2} w}{\partial \theta^{2}}+\frac{1}{r} \frac{\partial w}{\partial r}\right)$,

$N_{\theta \theta}^{i}=A_{12}^{i} \frac{\partial u}{\partial r}+A_{11}^{i}\left(\frac{1}{r} \frac{\partial v}{\partial \theta}+\frac{u}{r}\right)-B_{12}^{i} \frac{\partial^{2} w}{\partial r^{2}}-B_{11}^{i}\left(\frac{1}{r^{2}} \frac{\partial^{2} w}{\partial \theta^{2}}+\frac{1}{r} \frac{\partial w}{\partial r}\right)$,

$N_{r \theta}^{i}=A_{33}^{i}\left(\frac{1}{r} \frac{\partial u}{\partial \theta}+\frac{\partial v}{\partial r}-\frac{v}{r}\right)-B_{33}^{i}\left(\frac{2}{r} \frac{\partial^{2} w}{\partial r \partial \theta}-\frac{2}{r^{2}} \frac{\partial w}{\partial \theta}\right)$,

$M_{r r}^{i}=B_{11}^{i} \frac{\partial u}{\partial r}+B_{12}\left(\frac{1}{r} \frac{\partial v}{\partial \theta}+\frac{u}{r}\right)-D_{11}^{i} \frac{\partial^{2} w}{\partial r^{2}}-D_{12}^{i}\left(\frac{1}{r^{2}} \frac{\partial^{2} w}{\partial \theta^{2}}+\frac{1}{r} \frac{\partial w}{\partial r}\right)$,

$M_{\theta \theta}^{i}=B_{12}^{i} \frac{\partial u}{\partial r}+B_{11}^{i}\left(\frac{1}{r} \frac{\partial v}{\partial \theta}+\frac{u}{r}\right)-D_{12}^{i} \frac{\partial^{2} w}{\partial r^{2}}-D_{11}^{i}\left(\frac{1}{r^{2}} \frac{\partial^{2} w}{\partial \theta^{2}}+\frac{1}{r} \frac{\partial w}{\partial r}\right)$,

$M_{r \theta}^{i}=B_{33}^{i}\left(\frac{1}{r} \frac{\partial u}{\partial \theta}+\frac{\partial v}{\partial r}-\frac{v}{r}\right)-D_{33}^{i}\left(\frac{2}{r} \frac{\partial^{2} w}{\partial r \partial \theta}-\frac{2}{r^{2}} \frac{\partial w}{\partial \theta}\right)$.

In Eq. (14c) $\rho^{i}$ is the averaged material density of the FGM circular plate for the $i$-th distribution of porosities presented in the general form:

$\rho^{i} \equiv \rho^{i}(g, \psi)=\frac{1}{h} \int_{-h / 2}^{h / 2} \rho^{i}(z, g, \psi) d z, \quad i=\{e, u\}$.

Substituting Eqs. (15) and Eqs. (16) into Eqs. (14), and using relations given in Eq. (10), we get the coupled equilibrium equations of motion of the porous FGM circular plate presented in terms of displacement components:

$A_{11}^{i}\left(\frac{\partial^{2} u}{\partial r^{2}}+\frac{1}{r} \frac{\partial u}{\partial r}-\frac{u}{r^{2}}-\frac{1}{r^{2}} \frac{\partial v}{\partial \theta}+\frac{1}{r} \frac{\partial^{2} v}{\partial r \partial \theta}\right)+A_{33}^{i}\left(\frac{1}{r^{2}} \frac{\partial^{2} u}{\partial \theta^{2}}-\frac{1}{r} \frac{\partial^{2} v}{\partial r \partial \theta}-\frac{1}{r^{2}} \frac{\partial v}{\partial \theta}\right)-B_{11}^{i} \frac{\partial \nabla^{2} w}{\partial r}=0$,

$A_{11}^{i}\left(\frac{1}{r^{2}} \frac{\partial u}{\partial \theta}+\frac{1}{r} \frac{\partial^{2} u}{\partial r \partial \theta}+\frac{1}{r^{2}} \frac{\partial^{2} v}{\partial \theta^{2}}\right)+A_{33}^{i}\left(\frac{1}{r^{2}} \frac{\partial u}{\partial \theta}-\frac{1}{r} \frac{\partial^{2} u}{\partial r \partial \theta}+\frac{\partial^{2} v}{\partial r^{2}}+\frac{1}{r} \frac{\partial v}{\partial r}-\frac{v^{2}}{r}\right)-B_{11}^{i} \frac{1}{r} \frac{\partial \nabla^{2} w}{\partial \theta}=0$,

$D_{11}^{i} \nabla^{2} \nabla^{2} w-B_{11}^{i} \nabla^{2} \varepsilon=-\rho^{i} h \frac{\partial^{2} w}{\partial t^{2}}$,

where $\nabla^{2}=\frac{\partial^{2}}{\partial r^{2}}+\frac{1}{r} \frac{\partial}{\partial r}+\frac{1}{r^{2}} \frac{\partial^{2}}{\partial \theta^{2}}$ is the Laplace operator presented in polar coordinates and $\varepsilon=\frac{\partial u}{\partial r}+\frac{1}{r} \frac{\partial v}{\partial \theta}+\frac{u}{r}$.

\subsection{Decoupled equation of motion}

Eqs. (18) show, that the in-plane stretching and bending are coupled because the reference surface is a geometrical midplane. We can eliminate this coupling by introducing the physical neutral surface [26], where the in-plane displacements will be omitted. The in-plane displacements of the midplane can be expressed in terms of the slopes of deflection in the following form:

$u(r, \theta, t)=z_{0} \frac{\partial w(r, \theta, t)}{\partial r}$, 
$v(r, \theta, t)=z_{0} \frac{1}{r} \frac{\partial w(r, \theta, t)}{\partial \theta}$,

where $z_{0}$ is the distance between the midplane and the physical neutral surface. By substituting Eqs. (20) into Eqs. (6) and Eqs. (15) and letting $z=z_{0}$, the in-plane displacements $u, v$ and the in-plane forces $N_{r r}^{i}, N_{\theta \theta}^{i}, N_{r \theta}^{i}$ must equal zero based on properties of the physical neutral surface. By substituting Eqs. (20) into Eqs. (15) presented in the following form:

$N_{r r}^{i}=\left(z_{0} A_{11}^{i}-B_{11}^{i}\right) \frac{\partial^{2} w}{\partial r^{2}}+\left(z_{0} A_{12}^{i}-B_{12}^{i}\right)\left(\frac{1}{r^{2}} \frac{\partial^{2} w}{\partial \theta^{2}}+\frac{1}{r} \frac{\partial w}{\partial r}\right)=0$,

$N_{\theta \theta}^{i}=\left(z_{0} A_{12}^{i}-B_{12}^{i}\right) \frac{\partial^{2} w}{\partial r^{2}}+\left(z_{0} A_{11}^{i}-B_{11}^{i}\right)\left(\frac{1}{r^{2}} \frac{\partial^{2} w}{\partial \theta^{2}}+\frac{1}{r} \frac{\partial w}{\partial r}\right)=0$,

$N_{r \theta}^{i}=\left(z_{0} A_{33}^{i}-B_{33}^{i}\right)\left(\frac{2}{r} \frac{\partial^{2} w}{\partial r \partial \theta}-\frac{2}{r^{2}} \frac{\partial w}{\partial \theta}\right)=0$

and assuming that the Poisson's ratio is constant, distance $z_{0}$ can be obtained from relations:

$z_{0} A_{11}^{i}-B_{11}^{i}=z_{0} A_{12}^{i}-B_{12}^{i}=z_{0} A_{33}^{i}-B_{33}^{i}=0$,

where

$Z_{0}=\frac{B_{11}^{i}}{A_{11}^{i}}=\frac{B_{12}^{i}}{A_{12}^{i}}=\frac{B_{33}^{i}}{A_{33}^{i}}=\frac{\int_{-h / 2}^{h / 2} E^{i}(z, g, \psi) z d z}{\int_{-h / 2}^{h / 2} E^{i}(z, g, \psi) d z}$.

By substituting Eqs. (20) and Eq. (23) into Eq. (19) and Eq. (18c), we obtain the decoupled equation of transverse vibration of the porous FGM thin circular plate in the form:

$\mathfrak{D}^{i} \nabla^{2} \nabla^{2} w=-\rho^{i} h \frac{\partial^{2} w}{\partial t^{2}}$

where

$\mathfrak{D}^{i}=D_{11}^{i}-\frac{\left(B_{11}^{i}\right)^{2}}{A_{11}^{i}}$.

\section{Formulation of the problem}

For a harmonic solution, the non-axisymmetric deflection of the porous FGM circular plate may be expressed as follows:

$w(r, \theta, t)=W(r) \cos (n \theta) \cos (\omega t)$,

where $W(r)$ is the radial mode function as the small deflection compared with the thickness $h$ of the plate, $n$ is the integer number of diagonal nodal lines, $\theta$ is the angular coordinate, $\omega$ is the natural frequency. By substituting Eq. (26) into Eq. (24) using the dimensionless coordinate $\xi=r / R$ $(0<\xi \leq 1)$, the general governing differential equation assumes the following form:

$\mathcal{L}_{n}(W)=\rho^{i} h \omega^{2} W$

where $\mathcal{L}_{n}(\cdot)$ is the differential operator defined by

$$
\mathcal{L}_{n}(\cdot) \equiv \mathfrak{D}^{i} \frac{d^{4}}{d \xi^{4}}+\frac{2 \mathfrak{D}^{i}}{\xi} \frac{d^{3}}{d \xi^{3}}-\frac{\left(1+2 n^{2}\right) \mathcal{D}^{i}}{\xi^{2}} \frac{d^{2}}{d \xi^{2}}+\frac{\left(1+2 n^{2}\right) \mathcal{D}^{i}}{\xi^{3}} \frac{d}{d \xi}+\frac{\left(n^{4}-4 n^{2}\right) \mathfrak{D}^{i}}{\xi^{4}} .
$$

The calculated general forms of material density $\rho^{i}$ and the coefficients of extensional stiffness $\left(A_{11}^{i}\right)$, extensional-bending coupling stiffness $\left(B_{11}^{i}\right)$ and bending stiffness $\left(D_{11}^{i}\right)$ for the porous FGM circular plate are presented in the following general forms:

$\rho^{i}=\frac{\rho_{c}(2 x-\psi-g \psi)+\rho_{m}(2 x g-\psi-\psi g)}{2 x(1+g)}$,

$A_{11}^{i}=\frac{E_{c} h}{1-v^{2}}\left[\frac{(2 x-\psi-g \psi)+\frac{E_{m}}{E_{c}}(2 x g-\psi-g \psi)}{2 x(1+g)}\right]$, 


$$
\begin{aligned}
& B_{11}^{e}=B_{11}^{u}=\frac{E_{c} h^{2}}{\left(1-v^{2}\right)}\left[\frac{g\left(1-\frac{E_{m}}{E_{c}}\right)}{2(1+g)(2+g)}\right], \\
& D^{i}=\frac{E_{c} h^{3}}{12\left(1-v^{2}\right)}\left[\frac{y\left(6 g^{2}+6 g+12\right)-\psi(1+g)(2+g)(3+g)+\frac{E_{m}}{E_{c}}\left[y\left(2 g^{3}+6 g^{2}+16 g\right)-\psi(1+g)(2+g)(3+g)\right]}{2 y(1+g)(2+g)(3+g)}\right],
\end{aligned}
$$

where $x=y=1$ for the even distribution $(i=e)$ of porosities and $x=2, y=4$ for the uneven $(i=u)$ distribution of porosities. The extensional-bending coupling stiffness $B_{11}^{i}$ have the same form for both types of porosities.

By substituting the obtained forms from Eqs. (29) into Eq. (27), the generalized ordinary differential equation with variable coefficients is obtained as:

$\mathcal{L}_{n}(W)_{\chi}=\lambda^{2} \mu^{i} W$,

where

$$
\begin{aligned}
& \mathcal{L}_{n}(\cdot)_{\chi} \equiv\left(\chi_{1}^{i}+\chi_{2}^{i}\right) \frac{d^{4}}{d \xi^{4}}+\frac{2\left(\chi_{1}^{i}+\chi_{2}^{i}\right)}{\xi} \frac{d^{3}}{d \xi^{3}}-\frac{\left(1+2 n^{2}\right)\left(\chi_{1}^{i}+\chi_{2}^{i}\right)}{\xi^{2}} \frac{d^{2}}{d \xi^{2}} \\
& +\frac{\left(1+2 n^{2}\right)\left(\chi_{1}^{i}+\chi_{2}^{i}\right)}{\xi^{3}} \frac{d}{d \xi}+\frac{\left(n^{4}-4 n^{2}\right)\left(\chi_{1}^{i}+\chi_{2}^{i}\right)}{\xi^{4}} \\
& \chi_{1}^{i}=\frac{6 x g^{2}\left(E_{c}-E_{m}\right)^{2}}{E_{c}(1+g)(2+g)^{2}\left[E_{c}(\psi+g \psi-2 x)+E_{m}(\psi+g \psi-2 x g)\right]} \\
& \chi_{2}^{i}=\frac{E_{c}\left[y\left(12+6 g+6 g^{2}\right)-\psi(1+g)(2+g)(3+g)\right]+E_{m}\left[y\left(16 g+6 g^{2}+2 g^{3}\right)-\psi(1+g)(2+g)(3+g)\right]}{2 y E_{c}(1+g)(2+g)(3+g)} \\
& \mu^{i}=\frac{(-g \psi-\psi+2 x)-\frac{\rho_{m}}{\rho_{c}}(g \psi+\psi-2 x g)}{2 x(1+g)} \\
& \lambda=\omega R^{2} \sqrt{\rho_{c} h / D_{c}} \\
& D_{c}=\frac{E_{c} h^{3}}{12\left(1-v^{2}\right)}
\end{aligned}
$$

The boundary conditions on the outer edge $(\xi=1)$ of the porous FGM circular plate may be one of the following: clamped, simply supported, sliding supported and free. These conditions may be written in terms of the radial mode function $W(\xi)$ in the following form:

- Clamped:

$$
\begin{aligned}
& \left.W(\xi)\right|_{\xi=1}=0, \\
& \left.\frac{d W}{d \xi}\right|_{\xi=1}=0 .
\end{aligned}
$$

- Simply supported:

$\left.W(\xi)\right|_{\xi=1}=0$,

$\left.M(W)\right|_{\xi=1}=\left[\frac{d^{2} W}{d \xi^{2}}+\frac{v}{\xi} \frac{d W}{d \xi}-\frac{v n^{2}}{\xi^{2}} W\right]_{\xi=1}=0$.

- Sliding supported:

$\left.\frac{d W}{d \xi}\right|_{\xi=1}=0$,

$\left.V(W)\right|_{\xi=1}=\left[\frac{d^{3} W}{d \xi^{3}}+\frac{1}{\xi} \frac{d^{2} W}{d \xi^{2}}-\left(\frac{1+2 n^{2}-v n^{2}}{\xi^{2}}\right) \frac{d W}{d \xi}+\left(\frac{3 n^{2}-v n^{2}}{\xi^{3}}\right) W\right]_{\xi=1}=0$,

- Free:

$\left.M(W)\right|_{\xi=1}=0$,

$\left.V(W)\right|_{\xi=1}=0$. 
The static forces $M(W)$ and $V(W)$ are the normalized radial bending moment and the normalized effective shear force, respectively.

\section{Solution of the problem}

The one multiparametric general solution of the defined differential equation (30) for FGM circular/annular plates with the two various types of distribution of porosities $(i=\{e, u\})$ is obtained in the following form:

$W_{n}^{i}(\xi, \lambda, g, \psi)=C_{1} J_{n}\left[\left(\lambda \sqrt{\mathfrak{M}_{i}}\right)^{1 / 2} \xi\right]+C_{2} I_{n}\left[\left(\lambda \sqrt{\mathfrak{M}_{i}}\right)^{1 / 2} \xi\right]+C_{3} Y_{n}\left[\left(\lambda \sqrt{\mathfrak{M}_{i}}\right)^{1 / 2} \xi\right]+$

$C_{4} K_{n}\left[\left(\lambda \sqrt{\mathfrak{M}_{i}}\right)^{1 / 2} \xi\right]$,

where $n\left(n \in \mathbb{N}^{+}\right)$is the number of nodal lines, $C_{1}, C_{2}, C_{3}, C_{4}$ are the constants of integration, $J_{n}\left[\left(\lambda \sqrt{\mathfrak{M}_{i}}\right)^{1 / 2} \xi\right], I_{n}\left[\left(\lambda \sqrt{\mathfrak{M}_{i}}\right)^{1 / 2} \xi\right], Y_{n}\left[\left(\lambda \sqrt{\mathfrak{M}_{i}}\right)^{1 / 2} \xi\right], K_{n}\left[\left(\lambda \sqrt{\mathfrak{M}_{i}}\right)^{1 / 2} \xi\right]$ are the Bessel functions as particular solutions of Eq. (30), $\mathfrak{M}_{i}$ is the generalized multiparametric function defined as:

$\mathfrak{M}_{i} \equiv \mathfrak{M}_{i}\left(x, y, g, \psi, E_{m}, E_{c}, \rho_{m}, \rho_{c}\right)=\frac{\Omega_{1}^{i}}{\Omega_{2}^{i}+\Omega_{3}^{i}}, \quad \mathfrak{M}_{i} \geq 1 \quad \forall g \in[0, \infty] \wedge \forall \psi \in[0,1)$,

where

$\Omega_{1}^{i}=-E_{c} x(2+g)^{2}\left[\rho_{c}(g \psi+\psi-2 x)+\rho_{m}(g \psi+\psi-2 x g)\right]$,

$\Omega_{2}^{i}=\frac{12 x y g^{2}\left(E_{c}-E_{m}\right)^{2} \rho_{c}}{E_{c}(g \psi+\psi-2 x)+E_{m}(g \psi+\psi-2 x g)}$

$\Omega_{3}^{i}=\frac{(2+g) \rho_{c}\left[E_{c}\left[y\left(12+6 g+6 g^{2}\right)-\psi(1+g)(2+g)(3+g)\right]+E_{m}\left[y\left(16 g+6 g^{2}+2 g^{3}\right)-\psi(1+g)(2+g)(3+g)\right]\right]}{3+g}$.

The functions $J_{n}\left[\left(\lambda \sqrt{\mathfrak{M}_{i}}\right)^{1 / 2} \xi\right]$ and $I_{n}\left[\left(\lambda \sqrt{\mathfrak{M}_{i}}\right)^{1 / 2} \xi\right]$ are the limited linear independent solutions $\left(\lim _{\xi \rightarrow 0} J_{n}\left[\left(\lambda \sqrt{\mathfrak{M}_{i}}\right)^{1 / 2} \xi\right]<\infty, \lim _{\xi \rightarrow 0} I_{n}\left[\left(\lambda \sqrt{\mathfrak{M}_{i}}\right)^{1 / 2} \xi\right]<\infty\right)$ of Eq. (30) for the axisymmetric and nonaxisymmetric deflections at center $(\xi=0)$ of the porous FGM circular plate and diverse values of the physically justified parameters $\lambda, g$ and $\psi$. The particular solutions $Y_{n}\left[\left(\lambda \sqrt{\mathfrak{M}_{i}}\right)^{1 / 2} \xi\right]$ and $K_{n}\left[\left(\lambda \sqrt{\mathfrak{M}_{i}}\right)^{1 / 2} \xi\right]$ are unlimited $\left(\lim _{\xi \rightarrow 0} Y_{n}\left[\left(\lambda \sqrt{\mathfrak{M}_{i}}\right)^{1 / 2} \xi\right]=-\infty, \lim _{\xi \rightarrow 0} K_{n}\left[\left(\lambda \sqrt{\mathfrak{M}_{i}}\right)^{1 / 2} \xi\right]=\infty\right)$ for the deflection at center of the plate, then the general solution (41) for the porous FGM circular plate can be presented in the new form:

$W_{n}^{i}(\xi, \lambda, g, \psi)=C_{1} \Psi_{1}+C_{2} \Psi_{2}$,

where

$\Psi_{1} \equiv J_{n}\left[\left(\lambda \sqrt{\mathfrak{M}_{i}}\right)^{1 / 2} \xi\right]$,

$\Psi_{2} \equiv I_{n}\left[\left(\lambda \sqrt{\mathfrak{M}_{i}}\right)^{1 / 2} \xi\right]$.

By applying the general solution (44) and the boundary conditions $(37 \div 40)$ as well as assuming the existence of the non-trivial constants $C_{1}$ and $C_{2}$, the general non-linear multiparametric characteristic equations of the FGM circular plate with the two various types of distribution of porosities were obtained in the form:

- Clamped (C): 
$\Delta_{C}^{i}(\lambda, \mathrm{g}, \psi, n, x, y) \equiv\left|\begin{array}{cc}\Psi_{1} & \Psi_{2} \\ \frac{\partial \Psi_{1}}{\partial \xi} & \frac{\partial \Psi_{2}}{\partial \xi}\end{array}\right|_{\xi=1}=0$

- Simply supported (SS):

$\Delta_{S S}^{i}(\lambda, \mathrm{g}, \psi, n, x, y) \equiv\left|\begin{array}{cc}\Psi_{1} & \Psi_{2} \\ M\left[\Psi_{1}\right] & M\left[\Psi_{2}\right]\end{array}\right|_{\xi=1}=0$

- Sliding supported (S):

$\Delta_{S}^{i}(\lambda, \mathrm{g}, \psi, n, x, y) \equiv\left|\begin{array}{cc}\frac{\partial \Psi_{1}}{\partial \xi} & \frac{\partial \Psi_{2}}{\partial \xi} \\ V\left[\Psi_{1}\right] & V\left[\Psi_{2}\right]\end{array}\right|_{\xi=1}=0$

- Free (F):

$\Delta_{F}^{i}(\lambda, g, \psi, n, x, y) \equiv\left|\begin{array}{ll}M\left[\Psi_{1}\right] & M\left[\Psi_{2}\right] \\ V\left[\Psi_{1}\right] & V\left[\Psi_{2}\right]\end{array}\right|_{\xi=1}=0$

If $x=y=1$ is introduced to Eqs. (42) and (45), then the obtained characteristic equations (46) will be valid for the FGM circular plates with even $(i=e)$ distribution of porosities. If $x=2, y=4$ is introduced to Eqs. (42) and (45), than the obtained characteristic equations (46) will be valid for the FGM circular plates with uneven $(i=u)$ distribution of porosities.

The general solution for the perfect (without porosity) FGM circular plate can be obtained from Eq. (44) and presented in the following form:

$W_{n}(\xi, \lambda, g) \equiv \lim _{\psi \rightarrow 0} W_{n}^{i}(\xi, \lambda, g, \psi)=C_{1} \lim _{\psi \rightarrow 0} J_{n}\left[\left(\lambda \sqrt{\mathfrak{M}_{i}}\right)^{1 / 2} \xi\right]+C_{2} \lim _{\psi \rightarrow 0} I_{n}\left[\left(\lambda \sqrt{\mathfrak{M}_{i}}\right)^{1 / 2} \xi\right]$.

After calculations, the final form of general solution for the perfect FGM circular plate is expressed as

$W_{n}(\xi, \lambda, g)=J_{n}\left[(\lambda \sqrt{\mathfrak{Y}})^{1 / 2} \xi\right]+I_{n}\left[(\lambda \sqrt{\mathfrak{Y}})^{1 / 2} \xi\right]$

where

$\mathfrak{V}=\frac{E_{c}(2+g)^{2}(3+g)\left(E_{c}+g E_{m}\right)\left(\rho_{c}+g \rho_{m}\right)}{\rho_{c}(1+g)\left[12 E_{c}^{2}+\left(28 g+16 g^{2}+4 g^{3}\right) E_{c} E_{m}+\left(7 g^{2}+4 g^{3}+g^{4}\right) E_{m}^{2}\right]}$.

The general solution for the perfect FGM circular plate with negligible effect of the coupling in-plane and transverse displacements was obtained in previous paper [30] in the form:

$W_{n}(\xi, \lambda, g)=C_{1} J_{n}\left[(\lambda \sqrt{\mathfrak{P}})^{1 / 2} \xi\right]+C_{2} I_{n}\left[(\lambda \sqrt{\mathfrak{P}})^{1 / 2} \xi\right]$,

where

$\mathfrak{P}=\frac{E_{c}(2+g)(3+g)\left(\rho_{c}+g \rho_{m}\right)}{\rho_{c}\left[3 E_{c}\left(2+g+g^{2}\right)+E_{m}\left(8 g+3 g^{2}+g^{3}\right)\right]}$.

\section{Parametric study}

The every single fundamental and lower dimensionless frequencies of the free axisymmetric and non-axisymmetric vibrations of porous FGM circular plate were calculated for diverse values of the power-law index $g$, the porosity volume fraction $\psi$ and different boundary conditions using the Newton method aided by calculation software MATHEMATICA v11.

The Poisson's ratio is taken as $v=0.3$ and its variation is assumed to be negligible. In the present study, aluminum is taken as the metal and alumina is taken as the ceramic material. The values of Young's modulus and densities are taken as follows: $E_{m}=70 \mathrm{GPa}, E_{c}=380 \mathrm{GPa}, \rho_{m}=2702 \mathrm{~kg} / \mathrm{m}^{3}, \rho_{c}=$ $3800 \mathrm{~kg} / \mathrm{m}^{3}$. 


\subsection{Imperfect FGM circular plate}

The obtained numerical results for the first three dimensionless frequencies $\lambda=\omega R^{2} \sqrt{\rho_{c} h / D_{c}}$ of the axisymmetric $(n=0)$ and non-axisymmetric $(n=1)$ vibrations of the perfect $(\psi \rightarrow 0)$ homogeneous $(g \rightarrow 0)$ circular plate with various boundary conditions are presented in Table 1 and compared with the results obtained by Wu and Liu [36], Yalcin et al. [37], Zhou et al. [38], Duan et al. [39]. The obtained numerical results for the perfect homogeneous circular plate are in excellent agreement with those available in the literature.

Table 1. The dimensionless frequencies of the perfect homogeneous circular plate.

\begin{tabular}{|c|c|c|c|c|c|c|c|c|c|}
\hline & \multirow{3}{*}{$\lambda$} & \multicolumn{2}{|c|}{ Clamped } & \multicolumn{2}{|c|}{$\begin{array}{c}\text { Simply } \\
\text { supported }\end{array}$} & \multicolumn{2}{|c|}{$\begin{array}{c}\text { Sliding } \\
\text { supported }\end{array}$} & \multicolumn{2}{|c|}{ Free } \\
\hline & & \multicolumn{8}{|c|}{$n$} \\
\hline & & $\mathbf{0}$ & 1 & $\mathbf{0}$ & 1 & 0 & 1 & $\mathbf{0}$ & 1 \\
\hline \multirow{5}{*}{$\lambda_{0}$} & Present & 4.935 & 13.898 & 4.935 & 13.898 & 14.682 & 3.082 & 9.003 & 20.474 \\
\hline & [36] & 4.935 & 13.898 & 4.935 & 13.898 & 14.682 & 3.082 & 9.003 & 20.475 \\
\hline & [37] & 4.935 & 13.898 & 4.935 & 13.898 & - & - & 9.003 & 20.474 \\
\hline & [38] & 4.935 & 13.898 & 4.935 & 13.898 & - & - & 9.003 & 20.474 \\
\hline & [39] & 4.935 & 13.898 & 4.935 & 13.898 & - & - & 9.003 & 20.474 \\
\hline \multirow{5}{*}{$\lambda_{1}$} & Present & 29.720 & 48.478 & 4.935 & 13.898 & 49.218 & 28.398 & 38.443 & 59.812 \\
\hline & [36] & 29.720 & 48.479 & 29.720 & 48.478 & 49.218 & 28.399 & 38.443 & 59.812 \\
\hline & [37] & 29.720 & 48.478 & 29.720 & 48.479 & - & - & 38.443 & 59.811 \\
\hline & [38] & 29.719 & 48.478 & 29.720 & 48.478 & - & - & 38.443 & 59.811 \\
\hline & [39] & 29.720 & 48.478 & 29.719 & 48.478 & - & - & 38.443 & 59.812 \\
\hline \multirow{5}{*}{$\lambda_{2}$} & Present & 89.104 & 120.079 & 74.156 & 102.773 & 103.499 & 72.859 & 87.750 & 118.957 \\
\hline & [36] & 89.104 & 120.079 & 74.156 & 102.772 & 103.499 & 72.859 & 87.750 & 118.957 \\
\hline & [37] & 89.104 & 120.079 & 74.156 & 102.773 & - & - & 87.705 & 118.957 \\
\hline & [38] & 89.104 & 120.080 & 74.156 & 102.773 & - & - & 87.750 & 118.957 \\
\hline & [39] & 89.104 & 120.079 & 74.156 & 102.773 & - & - & 87.750 & 118.957 \\
\hline
\end{tabular}

The calculated fundamental dimensionless frequencies $\lambda_{0}$ of the axisymmetric $(n=0)$ and nonaxisymmetric $(n=1)$ vibrations of the FGM circular plate with evenly $(i=e)$ and unevenly $(i=u)$ distributed porosity are presented in Tables $2 \div 5$. In the parametric study, values of the power-law index of FGMs is taken as $g=\{0,0.2,0.4,0.6,1,2,3,4,5\}$ and values of the porosity volume fraction is taken as $\psi=\{0,0.05,0.1,0.2,0.3\}$.

The examples of dependences of the fundamental dimensionless frequencies $\lambda_{0}$ of the free axisymmetric $(n=0)$ vibrations of the circular plate on selected values of the power-law index and the porosities volume fraction are presented in Figures $3 \div 6$ for the two various types of distribution of porosity and the all considered boundary conditions.

\subsection{Perfect FGM circular plate}

The obtained general solution $(48)$ and the defined boundary conditions $(37 \div 40)$ were used to calculate the first three dimensionless frequencies $\lambda$ of the axisymmetric $(n=0)$ and non-axisymmetric $(n=1)$ vibrations of the perfect $(\psi=0)$ FGM circular plate with various boundary conditions. 
Table 2. The dimensionless fundamental frequencies of the clamped porous FGM circular plate.

\begin{tabular}{|c|c|c|c|c|c|c|c|c|c|c|c|}
\hline \multirow{3}{*}{$i$} & \multirow{3}{*}{$n$} & \multirow{3}{*}{$\psi$} & \multicolumn{9}{|c|}{$g$} \\
\hline & & & 0 & 0.2 & 0.4 & 0.6 & 1 & 2 & 3 & 4 & 5 \\
\hline & & & \multicolumn{9}{|c|}{$\lambda_{0}$} \\
\hline \multirow{10}{*}{$e$} & \multirow{5}{*}{0} & 0 & 10.215 & 9.481 & 8.896 & 8.436 & 7.797 & 7.090 & 6.867 & 6.777 & 6.724 \\
\hline & & 0.05 & 10.286 & 9.522 & 8.905 & 8.414 & 7.718 & 6.920 & 6.661 & 6.559 & 6.503 \\
\hline & & 0.1 & 10.362 & 9.566 & 8.914 & 8.387 & 7.623 & 6.712 & 6.401 & 6.280 & 6.219 \\
\hline & & 0.2 & 10.535 & 9.668 & 8.932 & 8.315 & 7.374 & 6.113 & 5.612 & 5.402 & 5.305 \\
\hline & & 0.3 & 10.745 & 9.792 & 8.948 & 8.207 & 6.993 & 5.034 & 3.949 & 3.312 & 2.923 \\
\hline & \multirow{5}{*}{1} & 0 & 21.260 & 19.731 & 18.514 & 17.557 & 16.228 & 14.756 & 14.292 & 14.105 & 13.993 \\
\hline & & 0.05 & 21.406 & 19.816 & 18.533 & 17.510 & 16.062 & 14.402 & 13.863 & 13.650 & 13.533 \\
\hline & & 0.1 & 21.564 & 19.909 & 18.552 & 17.454 & 15.866 & 13.968 & 13.222 & 13.069 & 12.942 \\
\hline & & 0.2 & 21.925 & 20.121 & 18.590 & 17.304 & 15.346 & 12.723 & 11.680 & 11.242 & 11.041 \\
\hline & & 0.3 & 22.362 & 20.380 & 18.622 & 17.081 & 14.554 & 10.478 & 8.220 & 6.894 & 6.084 \\
\hline \multirow{10}{*}{$u$} & \multirow{5}{*}{0} & 0 & 10.215 & 9.481 & 8.896 & 8.436 & 7.797 & 7.090 & 6.867 & 6.777 & 6.724 \\
\hline & & 0.05 & 10.288 & 9.544 & 8.949 & 8.478 & 7.819 & 7.079 & 6.844 & 6.751 & 6.698 \\
\hline & & 0.1 & 10.364 & 9.611 & 9.004 & 8.521 & 7.840 & 7.065 & 6.816 & 6.719 & 6.666 \\
\hline & & 0.2 & 10.523 & 9.751 & 9.120 & 8.612 & 7.882 & 7.023 & 6.738 & 6.630 & 6.577 \\
\hline & & 0.3 & 10.696 & 9.903 & 9.246 & 8.710 & 7.923 & 6.959 & 6.622 & 6.495 & 6.438 \\
\hline & \multirow{5}{*}{1} & 0 & 21.260 & 19.731 & 18.514 & 17.557 & 16.228 & 14.756 & 14.292 & 14.105 & 13.993 \\
\hline & & 0.05 & 21.411 & 19.864 & 18.624 & 17.644 & 16.272 & 14.733 & 14.244 & 14.050 & 13.940 \\
\hline & & 0.1 & 21.568 & 20.001 & 18.738 & 17.734 & 16.316 & 14.703 & 14.182 & 13.983 & 13.874 \\
\hline & & 0.2 & 21.901 & 20.293 & 18.980 & 17.923 & 16.404 & 14.617 & 14.023 & 13.798 & 13.688 \\
\hline & & 0.3 & 22.260 & 20.610 & 19.243 & 18.127 & 16.490 & 14.483 & 13.782 & 13.517 & 13.399 \\
\hline
\end{tabular}

Table 3. The dimensionless fundamental frequencies of the simply supported porous FGM circular plate.

\begin{tabular}{|c|c|c|c|c|c|c|c|c|c|c|c|}
\hline \multirow{3}{*}{$i$} & \multirow{3}{*}{$\boldsymbol{n}$} & \multirow{3}{*}{$\psi$} & \multicolumn{9}{|c|}{$g$} \\
\hline & & & 0 & 0.2 & 0.4 & 0.6 & 1 & 2 & 3 & 4 & 5 \\
\hline & & & \multicolumn{9}{|c|}{$\lambda_{0}$} \\
\hline \multirow{10}{*}{$e$} & \multirow{5}{*}{0} & 0 & 4.935 & 4.580 & 4.297 & 4.075 & 3.767 & 3.425 & 3.317 & 3.274 & 3.248 \\
\hline & & 0.05 & 4.969 & 4.600 & 4.302 & 4.064 & 3.728 & 3.343 & 3.218 & 3.168 & 3.141 \\
\hline & & 0.1 & 5.005 & 4.621 & 4.306 & 4.051 & 3.683 & 3.242 & 3.092 & 3.033 & 3.004 \\
\hline & & 0.2 & 5.089 & 4.670 & 4.315 & 4.017 & 3.562 & 2.953 & 2.711 & 2.609 & 2.563 \\
\hline & & 0.3 & 5.190 & 4.730 & 4.322 & 3.965 & 3.378 & 2.432 & 1.908 & 1.600 & 1.412 \\
\hline & \multirow{5}{*}{1} & 0 & 13.898 & 12.898 & 12.103 & 11.477 & 10.608 & 9.646 & 9.343 & 9.220 & 9.147 \\
\hline & & 0.05 & 13.993 & 12.954 & 12.115 & 11.446 & 10.500 & 9.415 & 9.062 & 8.923 & 8.847 \\
\hline & & 0.1 & 14.097 & 13.015 & 12.127 & 11.410 & 10.372 & 9.131 & 8.708 & 8.543 & 8.460 \\
\hline & & 0.2 & 14.333 & 13.153 & 12.152 & 11.312 & 10.032 & 8.317 & 7.635 & 7.349 & 7.218 \\
\hline & & 0.3 & 14.618 & 13.322 & 12.173 & 11.166 & 9.514 & 6.849 & 5.373 & 4.506 & 3.977 \\
\hline \multirow{10}{*}{$u$} & \multirow{5}{*}{0} & 0 & 4.935 & 4.580 & 4.297 & 4.075 & 3.767 & 3.425 & 3.317 & 3.274 & 3.248 \\
\hline & & 0.05 & 4.970 & 4.611 & 4.323 & 4.095 & 3.777 & 3.420 & 3.306 & 3.261 & 3.236 \\
\hline & & 0.1 & 5.006 & 4.643 & 4.349 & 4.116 & 3.787 & 3.413 & 3.292 & 3.246 & 3.220 \\
\hline & & 0.2 & 5.083 & 4.710 & 4.406 & 4.160 & 3.808 & 3.393 & 3.255 & 3.203 & 3.177 \\
\hline & & 0.3 & 5.167 & 4.784 & 4.467 & 4.207 & 3.828 & 3.362 & 3.199 & 3.137 & 3.110 \\
\hline & \multirow{5}{*}{1} & 0 & 13.898 & 12.898 & 12.103 & 11.477 & 10.608 & 9.646 & 9.343 & 9.220 & 9.147 \\
\hline & & 0.05 & 13.997 & 12.985 & 12.174 & 11.534 & 10.637 & 9.631 & 9.311 & 9.185 & 9.113 \\
\hline & & 0.1 & 14.099 & 13.075 & 12.249 & 11.592 & 10.666 & 9.611 & 9.273 & 9.141 & 9.069 \\
\hline & & 0.2 & 14.317 & 13.266 & 12.408 & 11.716 & 10.724 & 9.555 & 9.167 & 9.020 & 8.948 \\
\hline & & 0.3 & 14.551 & 13.473 & 12.580 & 11.850 & 10.780 & 9.468 & 9.009 & 8.836 & 8.759 \\
\hline
\end{tabular}


Table 4. The dimensionless fundamental frequencies of the porous FGM circular plate with sliding support.

\begin{tabular}{|c|c|c|c|c|c|c|c|c|c|c|c|}
\hline \multirow{3}{*}{$i$} & \multirow{3}{*}{$n$} & \multirow{3}{*}{$\psi$} & \multicolumn{9}{|c|}{$g$} \\
\hline & & & 0 & 0.2 & 0.4 & 0.6 & 1 & 2 & 3 & 4 & 5 \\
\hline & & & \multicolumn{9}{|c|}{$\lambda_{0}$} \\
\hline \multirow{10}{*}{$e$} & \multirow{5}{*}{0} & 0 & .682 & 3.626 & 2.785 & .124 & 11.206 & 10.190 & 9.870 & 9.740 & 9.663 \\
\hline & & 0.05 & 14.782 & 13.685 & 12.798 & 12.092 & 11.092 & 9.946 & 9.573 & 9.426 & 9.346 \\
\hline & & 0.1 & 14.892 & 13.749 & 12.811 & 12.053 & 10.956 & 9.646 & 9.199 & 9.025 & 8.938 \\
\hline & & 0.2 & 15.141 & 13.895 & 12.837 & 11.950 & 10.597 & 8.786 & 8.066 & 7.764 & 7.625 \\
\hline & & 0.3 & 15.442 & 14.074 & 12.860 & 11.796 & 10.051 & 7.236 & 5.676 & 4.761 & 4.201 \\
\hline & \multirow{5}{*}{1} & 0 & 3.082 & 2.860 & 2.684 & 2.545 & 2.352 & 2.139 & 2.072 & 2.045 & 2.029 \\
\hline & & 0.05 & 3.11 & 2.873 & 2.687 & 2.538 & 2.328 & 2.088 & 2.010 & 1.980 & 1.962 \\
\hline & & 0.1 & 3.126 & 2.886 & 2.690 & 2.530 & 2.300 & 2.025 & 1.931 & 1.894 & 1.876 \\
\hline & & 0.2 & 3 & 2.917 & 2.695 & 2.509 & 2.225 & 1.844 & 1.693 & 1.630 & 1.600 \\
\hline & & 0.3 & 242 & 2.954 & 2.700 & 2.476 & 2.110 & 1.519 & 1.191 & 0.999 & 0.882 \\
\hline \multirow{10}{*}{ u } & \multirow{5}{*}{0} & 0 & 14.682 & 13.626 & 12.785 & 12.124 & 11.206 & 10.190 & 9.870 & 9.740 & 9.663 \\
\hline & & 0.05 & 14.786 & 13.717 & 12.861 & 12.184 & 11.237 & 10.174 & 9.836 & 9.703 & 9.627 \\
\hline & & 0.1 & 14 & 13.812 & 12.940 & 12.246 & 11.268 & 10. & 9.795 & 9.656 & 9.581 \\
\hline & & 0.2 & 15 & 14.014 & 13.107 & 77 & 11.328 & 10.094 & 9.684 & 9.529 & 9.453 \\
\hline & & 0.3 & 15.372 & 14.233 & 13.289 & 12.518 & 11.388 & 10.002 & 9.518 & 9.334 & 9.253 \\
\hline & \multirow{5}{*}{1} & 0 & 3.082 & 2.860 & 2.684 & 2.545 & 2.352 & 2.139 & 2.072 & 2.045 & 2.029 \\
\hline & & 0.05 & 3.104 & 2.880 & 2.700 & 2.558 & 2.359 & 2.136 & 2.065 & 2.037 & 2.021 \\
\hline & & 0.1 & 3.127 & 2.890 & 2.716 & 2.571 & 2.365 & 2.131 & 2.056 & 2.027 & 2.011 \\
\hline & & 0.2 & 3.175 & 2.942 & 2.752 & 2.598 & 2.378 & 2.119 & 2.033 & 2.000 & 1.984 \\
\hline & & 0.3 & 3.227 & 2.988 & 2.790 & 2.628 & 2.391 & 2.100 & 1.998 & 1.960 & 1.942 \\
\hline
\end{tabular}

Table 5. The dimensionless fundamental frequencies of the free porous FGM circular plate.

\begin{tabular}{|c|c|c|c|c|c|c|c|c|c|c|c|}
\hline \multirow{3}{*}{$i$} & \multirow{3}{*}{$n$} & \multirow{3}{*}{$\psi$} & \multicolumn{9}{|c|}{$g$} \\
\hline & & & 0 & 0.2 & 0.4 & 0.6 & 1 & 2 & 3 & 4 & 5 \\
\hline & & & \multicolumn{9}{|c|}{$\lambda_{0}$} \\
\hline \multirow{10}{*}{$e$} & \multirow{5}{*}{0} & 0 & 9.003 & 8.355 & 7.840 & 7.435 & 6.872 & 6.248 & 6.052 & 5.973 & 5.926 \\
\hline & & 0.05 & 9.064 & 8.391 & 7.848 & 7.415 & 6.802 & 6.099 & 5.870 & 5.780 & 5.731 \\
\hline & & 0.1 & 9.132 & 8.431 & 7.856 & 7.391 & 6.718 & 5.915 & 5.641 & 5.534 & 5.480 \\
\hline & & 0.2 & 9.284 & 8.521 & 7.872 & 7.328 & 6.498 & 5.388 & 4.946 & 4.761 & 4.675 \\
\hline & & 0.3 & 9.469 & 8.630 & 7.886 & 7.233 & 6.163 & 4.437 & 3.481 & 2.919 & 2.576 \\
\hline & \multirow{5}{*}{1} & 0 & 20.474 & 19.002 & 17.830 & 16.908 & 15.628 & 14.211 & 13.764 & 13.584 & 13.476 \\
\hline & & 0.05 & 20.615 & 19.084 & 17.848 & 16.863 & 15.468 & 13.870 & 13.350 & 13.145 & 13.033 \\
\hline & & 0.1 & 20.767 & 19.173 & 17.866 & 16.809 & 15.280 & 13.452 & 12.829 & 12.586 & 12.464 \\
\hline & & 0.2 & 21.115 & 19.378 & 17.902 & 16.665 & 14.779 & 12.253 & 11.248 & 10.827 & 10.633 \\
\hline & & 0.3 & 21.535 & 19.628 & 17.934 & 16.450 & 14.016 & 10.091 & 7.916 & 6.639 & 5.859 \\
\hline \multirow{10}{*}{$u$} & \multirow{5}{*}{0} & 0 & 9.003 & 8.355 & 7.840 & 7.435 & 6.872 & 6.248 & 6.052 & 5.973 & 5.926 \\
\hline & & 0.05 & 9.067 & 8. & 7.886 & 7.471 & 6.890 & 6.239 & 6.032 & 5.950 & 5.903 \\
\hline & & 0.1 & 9.133 & 8.470 & 7.935 & 7.509 & 6.909 & 6.226 & 6.007 & 5.921 & 5.875 \\
\hline & & 0.2 & 9.274 & 8.593 & 8.037 & 7.590 & 6.947 & 6.190 & 5.938 & 5.843 & 5.796 \\
\hline & & 0.3 & 9.426 & 8.728 & 8.149 & 7.676 & 6.983 & 6.133 & 5.836 & 5.724 & 5.674 \\
\hline & \multirow{5}{*}{1} & 0 & 20.474 & 19.002 & 17.830 & 16.908 & 15.628 & 14.211 & 13.764 & 13.584 & 13.476 \\
\hline & & 0.05 & 20.620 & 19.129 & 17.935 & 16.992 & 15.670 & 14.188 & 13.718 & 13.531 & 13.425 \\
\hline & & 0.1 & 20.771 & 19.262 & 18.045 & 17.078 & 15.713 & 14.160 & 13.660 & 13.466 & 13.361 \\
\hline & & 0.2 & 21.091 & 19.543 & 18.279 & 17.261 & 15.798 & 14.077 & 13.505 & 13.288 & 13.182 \\
\hline & & 0.3 & 21.437 & 19.848 & 18.532 & 17.457 & 15.881 & 13.948 & 13.273 & 13.017 & 12.903 \\
\hline
\end{tabular}


a)

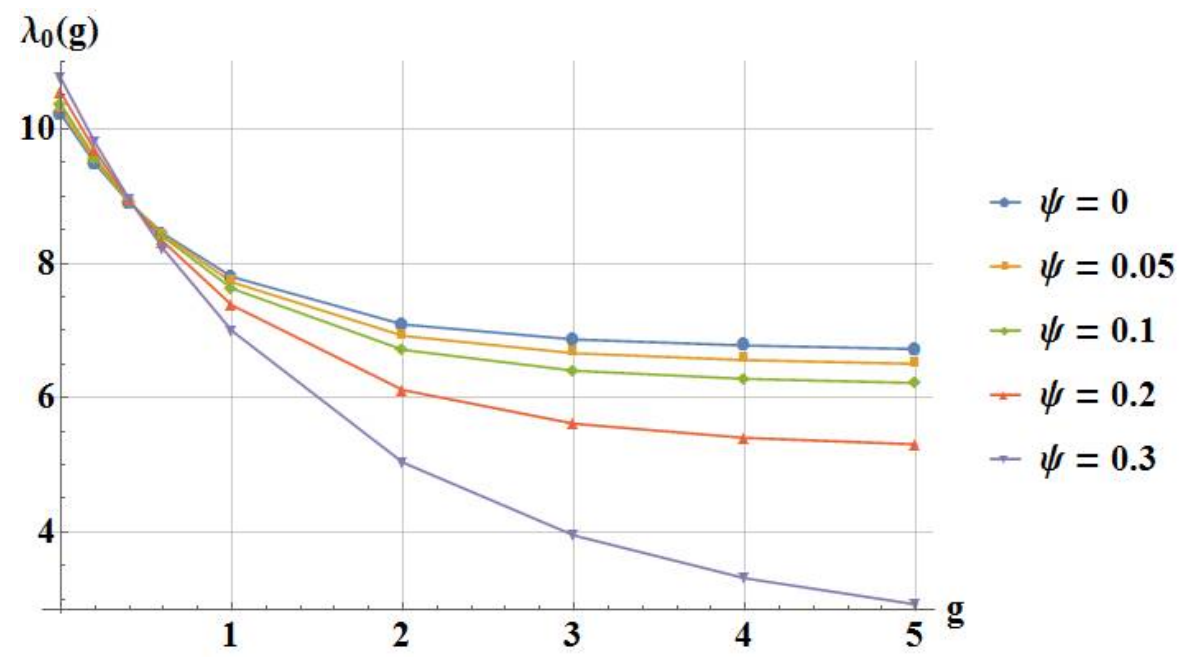

b)

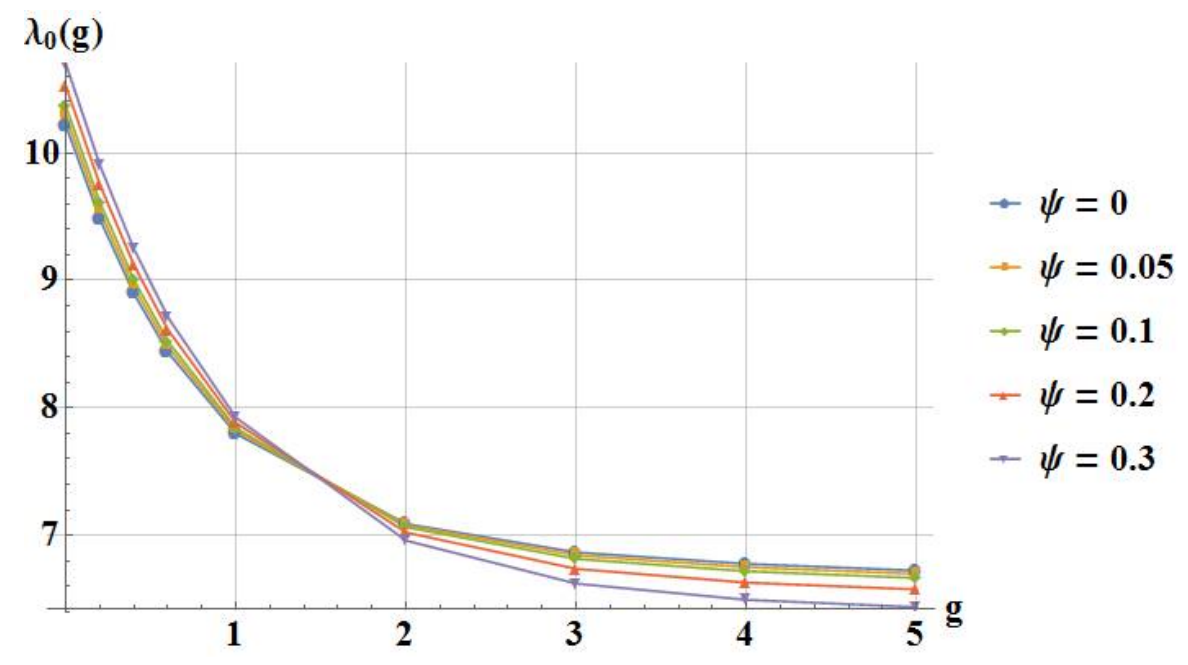

Fig. 3. The dependence of the fundamental dimensionless frequencies $\lambda_{0}$ of the free axisymmetric $(n=0)$ vibrations on selected values of the power-law index and the porosity volume fraction of the clamped circular plate with a) evenly and b) unevenly distributed porosities.

Table 6. The dimensionless frequencies of the clamped perfect FGM circular plate.

\begin{tabular}{|c|c|c|c|c|c|c|c|c|}
\hline \multirow{2}{*}{$n$} & & \multirow{2}{*}{$\lambda$} & \multicolumn{6}{|c|}{$\boldsymbol{g}$} \\
\hline & & & 1 & 2 & 3 & 4 & 5 & $\infty$ \\
\hline \multirow{9}{*}{0} & \multirow{3}{*}{$\lambda_{0}$} & Present & 7.797 & 7.090 & 6.867 & 6.777 & 6.724 & 5.199 \\
\hline & & [28] & 8.498 & 8.123 & 7.911 & 7.733 & 7.573 & - \\
\hline & & [30] & 8.498 & 8.123 & 7.911 & 7.733 & 7.573 & 5.199 \\
\hline & \multirow{3}{*}{$\lambda_{1}$} & Present & 30.357 & 27.604 & 26.737 & 26.386 & 26.177 & 20.243 \\
\hline & & [28] & 33.086 & 31.625 & 30.798 & 30.107 & 29.485 & - \\
\hline & & [30] & 33.086 & 31.625 & 30.798 & 30.107 & 29.485 & 20.243 \\
\hline & \multirow{3}{*}{$\lambda_{2}$} & Present & 68.012 & 61.845 & 59.902 & 59.116 & 58.649 & 45.352 \\
\hline & & [28] & 74.127 & 70.855 & 69.002 & 67.453 & 66.059 & - \\
\hline & & [30] & 74.127 & 70.855 & 69.002 & 67.453 & 66.059 & 45.352 \\
\hline \multirow{6}{*}{1} & \multirow{2}{*}{$\lambda_{0}$} & Present & 16.228 & 14.756 & 14.292 & 14.105 & 13.993 & 10.821 \\
\hline & & [30] & 17.687 & 16.906 & 16.464 & 16.094 & 15.762 & 10.821 \\
\hline & \multirow{2}{*}{$\lambda_{1}$} & Present & 46.430 & 42.219 & 40.893 & 40.357 & 40.038 & 30.961 \\
\hline & & [30] & 50.604 & 48.370 & 47.105 & 46.048 & 45.097 & 30.961 \\
\hline & \multirow{2}{*}{$\lambda_{2}$} & Present & 91.655 & 83.344 & 80.725 & 79.667 & 79.037 & 61.118 \\
\hline & & [30] & 99.896 & 95.486 & 92.989 & 90.901 & 89.024 & 61.118 \\
\hline
\end{tabular}


a)

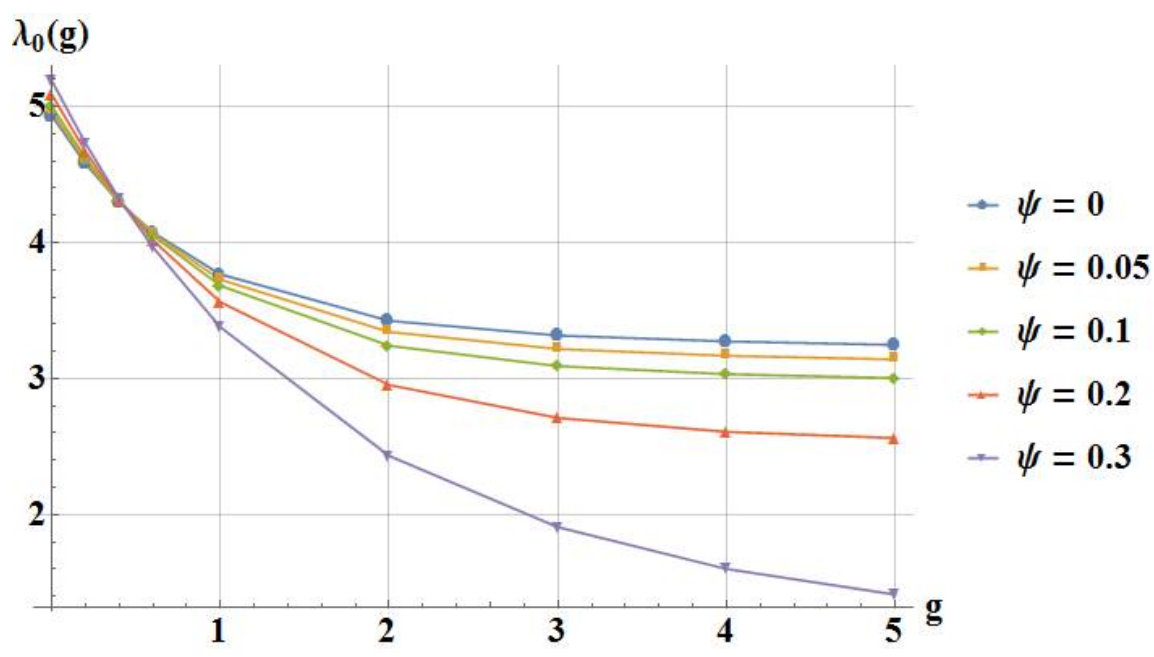

b)

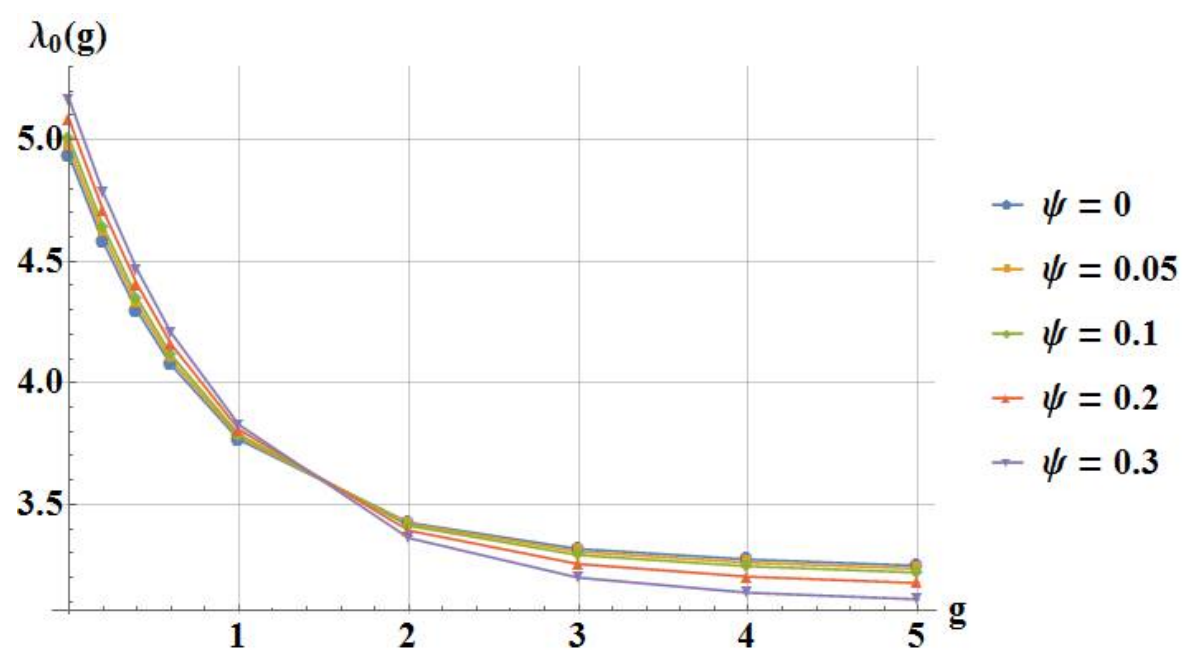

Fig. 4. The dependence of the fundamental dimensionless frequencies $\lambda_{0}$ of the free axisymmetric $(n=0)$ vibrations on selected values of the power-law index and the porosity volume fraction of the simply supported circular plate with a) evenly and b) unevenly distributed porosities.

Table 7. The dimensionless frequencies of the simply supported perfect FGM circular plate.

\begin{tabular}{|c|c|c|c|c|c|c|c|c|}
\hline & & \multirow{2}{*}{$\lambda$} & \multicolumn{6}{|c|}{$g$} \\
\hline$n$ & & & 1 & 2 & 3 & 4 & 5 & $\infty$ \\
\hline \multirow{8}{*}{0} & \multirow{3}{*}{$\lambda_{0}$} & Present & 3.767 & 3.425 & 3.317 & 3.274 & 3.248 & 2.512 \\
\hline & & [28] & 4.105 & 3.924 & 3.821 & 3.736 & 3.658 & - \\
\hline & & [30] & 4.105 & 3.924 & 3.821 & 3.736 & 3.658 & 2.512 \\
\hline & & Present & 22.685 & 20.628 & 19.980 & 19.717 & 19.562 & 15.127 \\
\hline & $\lambda_{1}$ & [28] & 24.724 & 23.633 & 23.015 & 22.498 & 22.033 & - \\
\hline & & [30] & 24.724 & 23.633 & 23.015 & 22.498 & 22.033 & 15.127 \\
\hline & & Present & 56.602 & 51.470 & 49.853 & 49.199 & 48.810 & 37.744 \\
\hline & $\lambda_{2}$ & [28] & 61.692 & 58.968 & 57.426 & 56.137 & 54.977 & - \\
\hline & & [30] & 61.692 & 58.968 & 57.426 & 56.137 & 54.977 & 37.744 \\
\hline \multirow{6}{*}{1} & & Present & 10.608 & 9.646 & 9.343 & 9.220 & $\begin{array}{l}9.147 \\
\end{array}$ & 7.074 \\
\hline & $\lambda_{0}$ & [30] & 11.562 & 11.051 & 10.762 & 10.521 & 10.303 & 7.074 \\
\hline & & Present & 37.003 & 33.648 & 32.591 & 32.163 & 31.909 & 24.675 \\
\hline & & [30] & 40.330 & 38.550 & 37.542 & 36.699 & 35.941 & 24.675 \\
\hline & & Present & 78.446 & 71.332 & 69.091 & 68.185 & 67.646 & 52.310 \\
\hline & $\lambda$ & [30] & 85.499 & 81.724 & 79.587 & 77.801 & 76.194 & 52.310 \\
\hline
\end{tabular}


a)

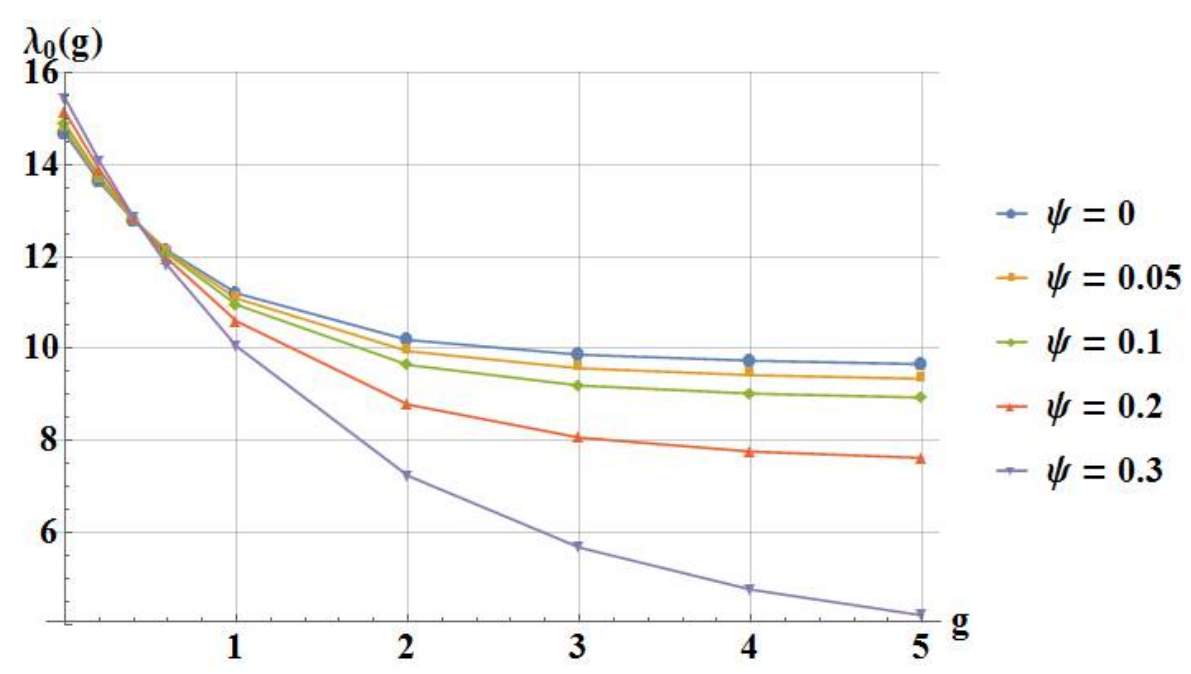

b)

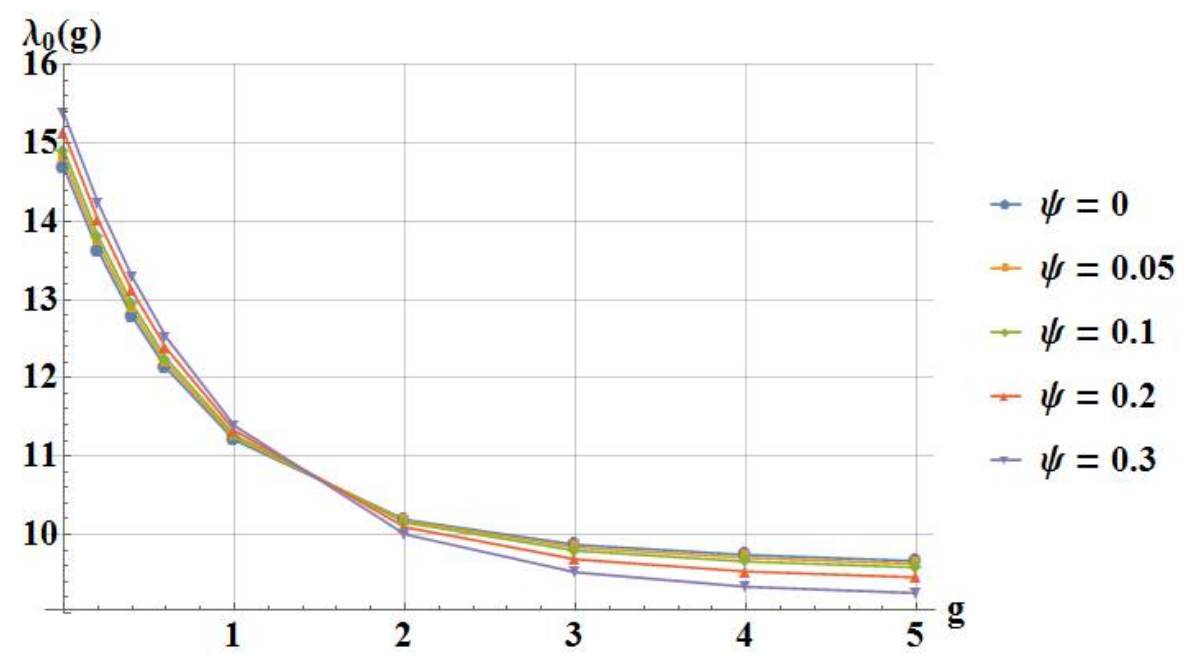

Fig. 5. The dependence of the fundamental dimensionless frequencies $\lambda_{0}$ of the free axisymmetric $(n=0)$ vibrations on selected values of the power-law index and the porosity volume fraction of the sliding supported circular plate with a) evenly and b) unevenly distributed porosities.

The obtained numerical results are presented in Tables $6 \div 9$ for selected values of the power-law index $g$. Numerical results obtained for the clamped and simply supported plates (Table 6 and Table 7) were compared with the results presented in previous papers $[28,30]$, where effect of the coupling inplane and transverse displacements was omitted.

The fundamental dimensionless frequencies of the perfect FGM circular plates with and without effect of the coupling in-plane and transverse displacements obtained for selected values of the powerlaw index and diverse boundary conditions are presented in Table 10. Additionally, the differences (errors) between obtained results were calculated according to the equation:

$\delta(\%)=\left(\frac{\lambda_{0}^{\Re}-\lambda_{0}^{\mathfrak{Y}}}{\lambda_{0}^{\Re}}\right) \cdot 100 \%$,

where $\lambda_{0}^{\mathfrak{P}}$ and $\lambda_{0}^{\mathfrak{Y}}$ are the fundamental dimensionless frequencies of the perfect FGM circular plate without and with effect of the coupling in-plane and transverse displacements, respectively. Figure 7 presents the dependence of the differences (errors) between obtained results for the power-law index $g \geq 0$. 
a)

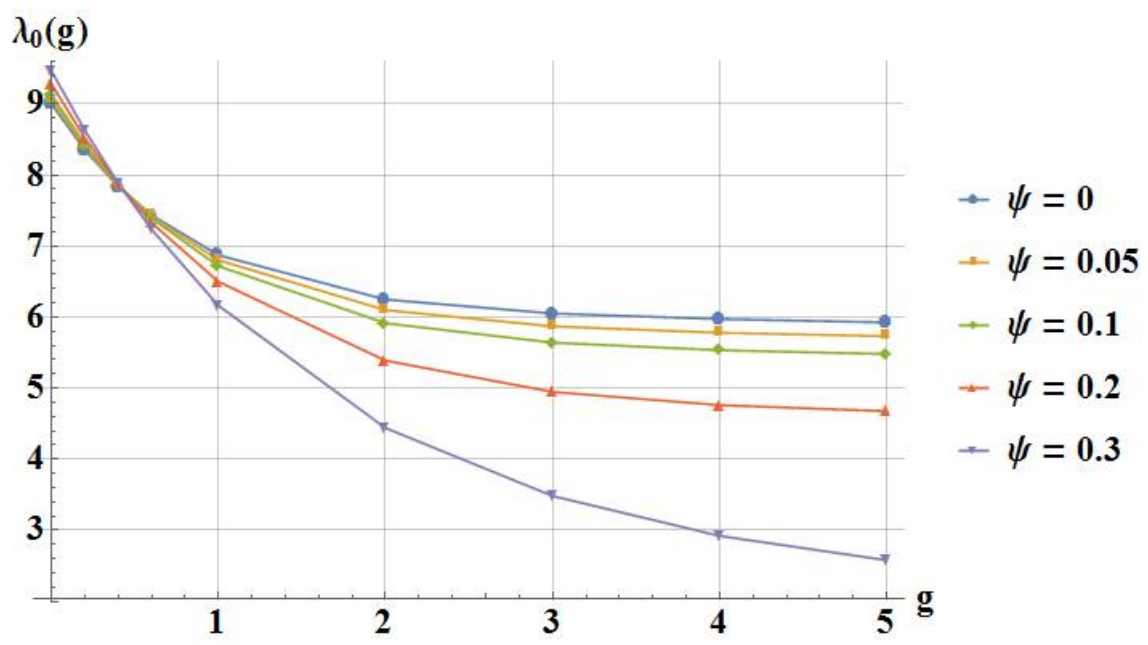

b)

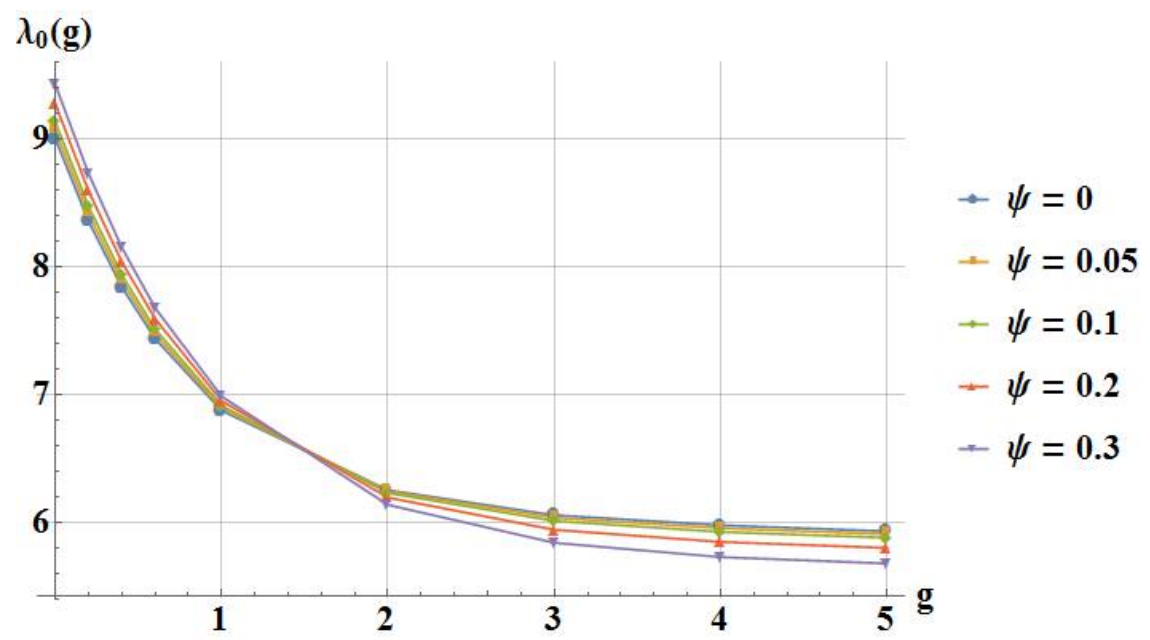

Fig. 6. The dependence of the fundamental dimensionless frequencies $\lambda_{0}$ of the free axisymmetric $(n=0)$ vibrations on selected values of the power-law index and the porosity volume fraction of the free circular plate with a) evenly and b) unevenly distributed porosities.

Table 8. The dimensionless frequencies of the free perfect FGM circular plate.

\begin{tabular}{cccccccc}
\hline \multirow{n}{n}{} & & $\boldsymbol{\lambda}$ & \multicolumn{6}{c}{$\boldsymbol{g}$} \\
\cline { 2 - 8 } & & $\mathbf{1}$ & $\mathbf{2}$ & $\mathbf{3}$ & $\mathbf{4}$ & $\mathbf{5}$ & $\infty$ \\
\hline \multirow{2}{*}{0} & $\lambda_{0}$ & 6.872 & 6.248 & 6.052 & 5.973 & 5.926 & 4.582 \\
& $\lambda_{1}$ & 29.343 & 26.682 & 25.844 & 25.505 & 25.303 & 19.567 \\
& $\lambda_{2}$ & 66.979 & 60.905 & 58.992 & 58.218 & 57.757 & 44.663 \\
\hline \multirow{2}{*}{1} & $\lambda_{0}$ & 15.628 & 14.211 & 13.764 & 13.584 & 13.476 & 10.421 \\
& $\lambda_{1}$ & 45.653 & 41.513 & 40.209 & 39.682 & 39.368 & 30.443 \\
& $\lambda_{2}$ & 90.799 & 82.565 & 79.971 & 78.922 & 78.298 & 60.547 \\
\hline
\end{tabular}

Table 9. The dimensionless frequencies of the perfect FGM circular plate with sliding support.

\begin{tabular}{cccccccc}
\hline \multirow{2}{n}{} & \multirow{\lambda}{\lambda}{} & \multicolumn{7}{c}{$\boldsymbol{g}$} \\
\cline { 2 - 8 } & $\mathbf{1}$ & $\mathbf{2}$ & $\mathbf{3}$ & $\mathbf{4}$ & $\mathbf{5}$ & $\infty$ \\
\hline \multirow{2}{*}{0} & $\lambda_{0}$ & 11.206 & 10.190 & 9.870 & 9.740 & 9.663 & 7.473 \\
& $\lambda_{1}$ & 37.568 & 34.161 & 33.088 & 32.654 & 32.396 & 25.051 \\
& $\lambda_{2}$ & 79.000 & 71.836 & 69.579 & 68.667 & 68.124 & 52.680 \\
\hline & $\lambda_{0}$ & 2.352 & 2.139 & 2.072 & 2.045 & 2.029 & 1.568 \\
1 & $\lambda_{1}$ & 21.676 & 19.711 & 19.091 & 18.841 & 18.692 & 14.454 \\
& $\lambda_{2}$ & 55.612 & 50.569 & 48.981 & 48.338 & 47.956 & 37.084 \\
\hline
\end{tabular}




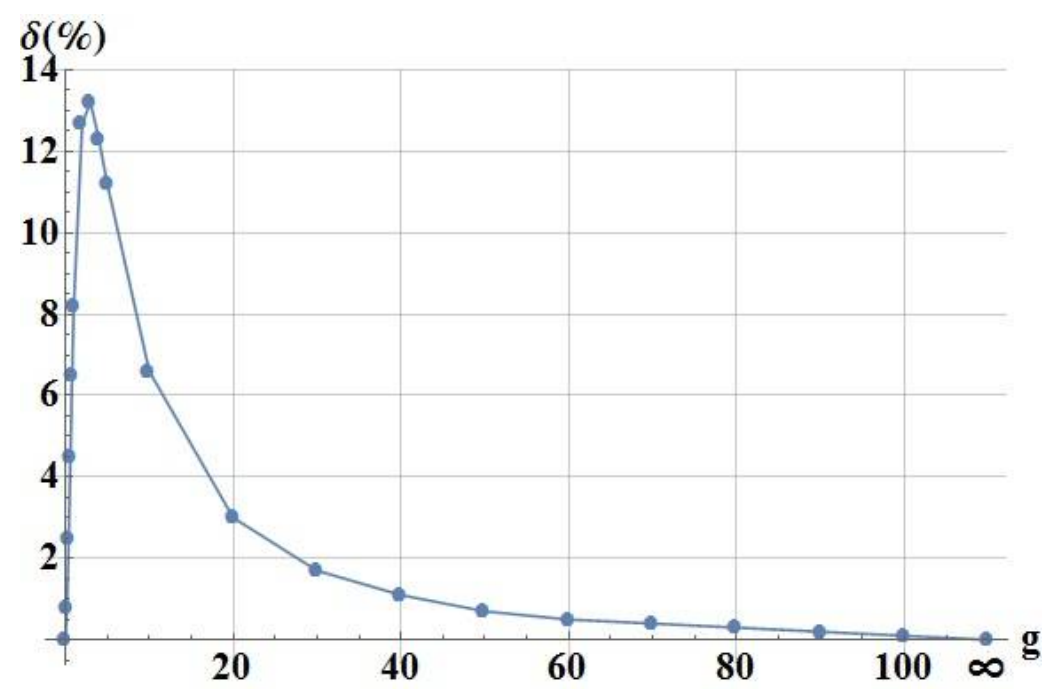

Fig. 7. The dependence of the differences (errors) between the fundamental dimensionless frequencies of the perfect FGM circular plate without $\left(\lambda_{0}^{\Re}\right)$ and with $\left(\lambda_{0}^{\mathfrak{Y}}\right)$ effect of the coupling in-plane and transverse displacements for diverse values of the power-law index.

Table 10. The differences between the fundamental dimensionless frequencies of the perfect FGM circular plates with and without effect of the coupling in-plane and transverse displacements.

\begin{tabular}{|c|c|c|c|c|c|c|c|c|c|c|c|}
\hline \multirow{2}{*}{ BCs } & \multirow{2}{*}{$n$} & \multirow{2}{*}{$\lambda$} & \multicolumn{9}{|c|}{$y$} \\
\hline & & & 1 & 2 & 3 & 4 & 5 & 10 & 30 & 60 & $\infty$ \\
\hline \multirow{6}{*}{ 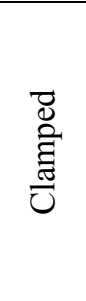 } & & $\lambda_{0}^{\mathfrak{P}}$ & 8.498 & 8.123 & 7.911 & 7.733 & 7.573 & 6.977 & 6.064 & 5.687 & 5.199 \\
\hline & 0 & $\lambda_{0}^{\mathfrak{Y}}$ & 7.797 & 7.090 & 6.867 & 6.777 & 6.724 & 6.512 & 5.960 & 5.654 & 5.199 \\
\hline & & $\boldsymbol{\delta}(\%)$ & 8.2 & 12.7 & 13.2 & 12.3 & 11.2 & 6.6 & 1.7 & 0.5 & 0 \\
\hline & & $\lambda_{0}^{\Re}$ & 17.687 & 16.906 & 16.464 & 16.094 & 15.762 & 14.520 & 12.621 & 11.835 & 10.821 \\
\hline & 1 & $\lambda_{0}^{\mathfrak{Z}}$ & 16.228 & 14.756 & 14.292 & 14.105 & 13.993 & 13.552 & 12.404 & 11.767 & 10.821 \\
\hline & & $\boldsymbol{\delta}(\%)$ & 8.2 & 12.7 & 13.2 & 12.3 & 11.2 & 6.6 & 1.7 & 0.5 & 0 \\
\hline \multirow{6}{*}{ 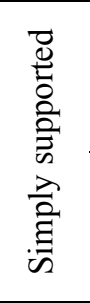 } & & $\lambda_{0}^{\mathfrak{P}}$ & 4.105 & 3.924 & 3.821 & 3.736 & 3.658 & 3.370 & 2.929 & 2.747 & 2.512 \\
\hline & 0 & $\lambda_{0}^{\mathfrak{Y}}$ & 3.767 & 3.425 & 3.317 & 3.274 & 3.248 & 3.145 & 2.879 & 2.731 & 2.512 \\
\hline & & $\delta(\%)$ & 8.2 & 12.7 & 13.2 & 12.3 & 11.2 & 6.6 & 1.7 & 0.5 & 0 \\
\hline & & $\lambda_{0}^{\mathfrak{P}}$ & 11.562 & 11.051 & 10.762 & 10.521 & 10.303 & 9.492 & 8.250 & 7.737 & 7.074 \\
\hline & 1 & $\lambda_{0}^{\mathfrak{Y}}$ & 10.608 & 9.646 & 9.343 & 9.220 & 9.147 & 8.859 & 8.108 & 7.692 & 7.074 \\
\hline & & $\boldsymbol{\delta}(\%)$ & 8.2 & 12.7 & 13.2 & 12.3 & 11.2 & 6.6 & 1.7 & 0.5 & 0 \\
\hline \multirow{6}{*}{ 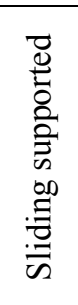 } & & $\lambda_{0}^{\mathfrak{P}}$ & 12.214 & 11.675 & 11.369 & 11.114 & 10.885 & 10.027 & 8.716 & 8.173 & 7.473 \\
\hline & 0 & $\lambda_{0}^{\mathfrak{Y}}$ & 11.206 & 10.190 & 9.870 & 9.740 & 9.663 & 9.359 & 8.566 & 8.126 & 7.473 \\
\hline & & $\delta(\%)$ & 8.2 & 12.7 & 13.2 & 12.3 & 11.2 & 6.6 & 1.7 & 0.5 & 0 \\
\hline & & $\lambda_{0}^{\mathfrak{P}}$ & 2.564 & 2.451 & 2.387 & 2.333 & 2.285 & 2.105 & 1.830 & 1.716 & 1.569 \\
\hline & 1 & $\lambda_{0}^{\mathfrak{Y}}$ & 2.352 & 2.139 & 2.072 & 2.045 & 2.029 & 1.964 & 1.798 & 1.706 & 1.569 \\
\hline & & $\boldsymbol{\delta}(\%)$ & 8.2 & 12.7 & 13.2 & 12.3 & 11.2 & 6.6 & 1.7 & 0.5 & 0 \\
\hline \multirow{6}{*}{ 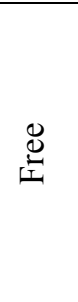 } & & $\lambda_{0}^{\mathfrak{P}}$ & 7.489 & 7.159 & 6.972 & 6.815 & 6.674 & 6.149 & 5.344 & 5.012 & 4.582 \\
\hline & 0 & $\lambda_{0}^{\mathfrak{V}}$ & 6.872 & 6.248 & 6.052 & 5.973 & 5.926 & 5.739 & 5.252 & 4.983 & 4.582 \\
\hline & & $\delta(\%)$ & 8.2 & 12.7 & 13.2 & 12.3 & 11.2 & 6.6 & 1.7 & 0.5 & 0 \\
\hline & & $\lambda_{0}^{\Re}$ & 17.033 & 16.281 & 15.855 & 15.499 & 15.179 & 13.984 & 12.155 & 11.398 & 10.421 \\
\hline & 1 & $\lambda_{0}^{\mathfrak{Y}}$ & 15.628 & 14.211 & 13.764 & 13.584 & 13.476 & 13.051 & 11.945 & 11.332 & 10.421 \\
\hline & & $\boldsymbol{\delta}(\%)$ & 8.2 & 12.7 & 13.2 & 12.3 & 11.2 & 6.6 & 1.7 & 0.5 & 0 \\
\hline
\end{tabular}




\section{Discussion}

\subsection{Imperfect FGM circular plate}

The numerical results for the fundamental dimensionless frequencies of the porous FGM circular plates presented in Tables $(2 \div 5)$ show the following dependences:

- the fundamental eigenfrequency $\lambda_{0}$ of the axisymmetric and non-axisymmetric vibrations of the circular plate decreases with the increasing value of the power-law index $g$ for the two considered distributions of porosities and all considered values of the porosity volume fraction $\psi$;

- for the evenly distributed porosities, the fundamental eigenfrequency $\lambda_{0}$ of the axisymmetric and non-axisymmetric vibrations of the plate increases with the increasing value of the porosity volume fraction $\psi$ for $g \in[0,0.4]$ and decreases for $g \in[0.6,5]$;

- for the unevenly distributed porosities, the fundamental eigenfrequency $\lambda_{0}$ of the axisymmetric and non-axisymmetric vibrations of the plate increases with the increasing value of the porosity volume fraction $\psi$ for $g \in[0,1]$ and decreases for $g \in[2,5]$;

- the influence of values of the porosity volume fraction $\psi$ on the values of the fundamental eigenfrequency $\lambda_{0}$ of the axisymmetric and non-axisymmetric vibrations of the plate is smaller for the unevenly distributed porosities than for the evenly distributed porosities;

- for the evenly distributed porosities, the fundamental eigenfrequency $\lambda_{0}$ of the axisymmetric and non-axisymmetric vibrations of plate decreases faster for $\psi=0.3$ with the increasing values of the power-law index $g$ than for $\psi=\{0,0.1,0.2\}$;

- for the unevenly distributed porosities, the fundamental eigenfrequency $\lambda_{0}$ of the axisymmetric and non-axisymmetric vibrations of the plate decreases slowly with the increasing values of the powerlaw index $g$ for all considered values of the porosity volume fraction $\psi$.

The observed dependences exist because of the diverse influence of porosity distributions, values of the power-law index and the porosity volume fraction on decreasing (increasing) the ratios of mass to stiffness of the considered circular plates. The all observed dependences are independent of the considered boundary conditions which influence only the values of the dimensionless frequencies of the plate.

\subsection{Perfect FGM circular plate}

It can be observed that the values of dimensionless frequencies of the perfect FGM circular plates obtained by omitting the effect of coupling in-plane and transverse displacements are higher than the values of the dimensionless frequencies of the considered plate with the coupling effect. The differences (errors) between the calculated dimensionless frequencies of free axisymmetric and non-axisymmetric vibration of the perfect FGM circular plate with and without the coupling effect are significant for the power-law index $g \in[0,20]$, but for $g \in[20, \infty]$ these differences decrease from $2 \%$ to $0 \%$. It can be observed from Table 10 that the differences between the calculated dimensionless frequencies are independent of the modes of vibration and the boundary conditions of the considered circular plate.

\section{Conclusions}

This paper presents the influence of two different types of distribution of porosities on the free vibrations of the thin functionally graded circular plate with clamped, simply supported, sliding supported, and free edges. To this aim the boundary value problem was formulated and a solution was obtained in the exact closed form. The universal multiparametric characteristic equations were defined using the properties of the universal multiparametric general solution obtained for the plate with even 
and uneven distribution of porosities. The effect of the power-law index, the volume fraction index and diverse boundary conditions on the values of the dimensionless frequencies of the free axisymmetric and non-axisymmetric vibrations of the circular plate was comprehensively studied. Additionally, the effect of the power-law index and different boundary conditions on the values of dimensionless frequencies of the FGM circular plate without porosities was also presented. The obtained general solution of the differential equation will allow to study the influences of diverse additional complicating effects such as stepped thickness, cracks, additional elements expressed by only additional boundary conditions on the dynamic behavior of the perfect and porous functionally graded circular plates.

This comprehensive investigation can be applied to predict the dynamic behavior of circular plates and to shape their dynamics in order to avoid resonance. The exact frequencies of vibration presented in non-dimensional form can serve as benchmark values for researchers and engineers to validate their analytical and numerical methods applied in design and analysis of porous functionally graded structural elements.

\section{Acknowledgements}

The research was conducted within S/WZ/1/2017 project and was financed by the funds of the Ministry of Science and Higher Education, Poland.

\section{References}

1. Zhu J, Lai Z, Yin Z, Jeon J, Lee S. Fabrication of $\mathrm{ZrO}_{2}-\mathrm{NiCr}$ functionally graded material by powder metallurgy. Materials Chemistry and Physics 2001;68:130-135.

2. Wattanasakulpong N, Prusty BG, Kelly DW, Hoffman M. Free vibration analysis of layered functionally graded beams with experimental validation. Materials and Design 2012;36:182-190.

3. Jabbari M, Farzaneh Joubaneh E, Khorshidvand AR, Eslami MR. Buckling analysis of porous circular plate with piezoelectric actuator layers under uniform radial compression. International Journal of Mechanical Sciences 2013;70:50-56.

4. Khorshidvand AR, Farzaneh Joubaneh E, Jabbari M, Eslami MR. Buckling analysis of a porous circular plate with piezoelectric sensor-actuator layers under uniform radial compression. Acta Mechanica 2014;225:179193.

5. Mojahedin A, Farzaneh Joubaneh E, Jabbari M. Thermal and mechanical stability of a circular porous plate with piezoelectric actuators. Acta Mechanica 2014;225:3437-3452.

6. Behravan Rad A, Shariyat M. Three-dimensional magneto-elastic analysis of asymmetric variable thickness porous FGM circular plates with non-uniform tractions and Kerr elastic foundations. Composite Structures 2015;125:558-574.

7. Barati MR, Sadr MH, Zenkour AM. Buckling analysis of higher order graded smart piezoelectric plates with porosities resting on elastic foundation. International Journal of Mechanical Sciences 2016;117:309-320.

8. Mechab I, Mechab B, Benaissa S, Serier B, Bachir Bouiadjra B. Free vibration analysis of FGM nanoplate with porosities resting on Winkler Pasternak elastic foundations based on two-variable refined plate theories. Journal of the Brazilian Society of Mechanical Sciences and Engineering 2016;38:2193-2211.

9. Mojahedin A, Jabbari M, Khorshidvand AR, Eslami MR. Buckling analysis of functionally graded circular plates made of saturated porous materials based on higher order shear deformation theory. Thin-Walled Structures 2016;99:83-90.

10. Wang YQ, Zu JW. Vibrations behaviors of functionally graded rectangular plates with porosities and moving in thermal environment. Aerospace Science and Technology 2017;69:550-562.

11. Gupta A, Talha M. Influence of porosity on the flexural and vibration response of gradient plate using nonpolynomial higher-order shear and normal deformation theory. International Journal of Mechanics and Materials in Design 2017. https://doi.org/10.1007/s10999-017-9369-2

12. Wang YQ, Zu JW. Vibration characteristics of moving sigmoid functionally graded plates containing porosities. International Journal of Mechanics and Materials in Design 2017. https://doi.org/10.1007/s10999$\underline{017-9385-2}$

13. Ebrahimi F, Jafari A, Barati MR. Free vibration analysis of smart porous plates subjected to various physical fields considering neutral surface position. Arabian Journal for Science and Engineering 2017;42:1865-1881. 
14. Feyzi MR, Khorshidvand AR. Axisymmetric post-buckling behavior of saturated porous circular plates. ThinWalled Structures 2017;112:149-158.

15. Wang YQ, Zu JW. Large-amplitude vibration of sigmoid functionally graded thin plates with porosities. ThinWalled Structures 2017;119:911-924.

16. Wang YQ, Wan YH, Zhang YF. Vibrations of longitudinally travelling functionally graded material plates with porosities. European Journal of Mechanics A/Solids 2017;66:55-68.

17. Ebrahimi F, Jafari A, Barati MR. Vibration analysis of magneto-electro-elastic heterogeneous porous material plates resting on elastic foundations. Thin-Walled Structures 2017;119:33-46.

18. Shahverdi H, Barati MR. Vibration analysis of porous functionally graded nanoplates. International Journal of Engineering Science 2017;120:82-99.

19. Shojaeefard MH, Googarchin HS, Ghadiri M, Mahinzare M. Micro temperature-dependent FG porous plate: Free vibration and thermal buckling analysis using modified couple stress theory with CPT and FSDT. Applied Mathematical Modelling 2017;50:633-655.

20. Barati MR, Shahverdi H. Nonlinear vibration of nonlocal four-variable graded plates with porosities implementing homotopy perturbation and Hamiltonian methods. Acta Mechanica 2018;229:343-362.

21. Kiran MC, Kattimani SC, Vinyas M. Porosity influence on structural behaviour of skew functionally graded magneto-electro-elastic plate. Composite Structures 2018;191:36-77.

22. Cong PH, Chien TM, Khoa ND, Duc ND. Nonlinear thermomechanical buckling and post-buckling response of porous FGM plates using Reddy's HSDT. Aerospace Science and Technology 2018;77:419-428.

23. Kiran MC, Kattimani SC. Assessment of porosity influence on vibration and static behaviour of functionally graded magneto-electro-elastic plate: A finite element study. European Journal of Mechanics A/Solids 2018;71:258-277.

24. Arshid E, Khorshidvand AR. Free vibration analysis of saturated porous FG circular plates integrated with piezoelectric actuators via differential quadrature method. Thin-Walled Structures 2018;125:220-233.

25. Shahsavari D, Shahsavari M, Li L, Karami B. A novel quasi-3D hyperbolic theory for free vibration of FG plates with porosities resting on Winkler/Pasternak/Kerr foundation. Aerospace Science and Technology 2018;72:134-149.

26. Zhang D-G, Zhou Y-H. A theoretical analysis of FGM thin plates based on physical neutral surface. Computational Materials Science 2008;44:716-720.

27. Ebrahimi F, Rastgo A. An analytical study on the free vibration of smart circular thin FGM plate based on classical plate theory. Thin-Walled Structures 2008;46:1402-1408.

28. Lal R, Ahlawat N. Axisymmetric vibrations and buckling analysis of functionally graded circular plates via differential transform method. European Journal of Mechanics A/Solids 2015;52:85-94.

29. Lal R, Ahlawat N. Buckling and vibration of functionally graded non-uniform circular plates resting on Winkler foundation. Latin American Journal of Solids and Structures 2015;12:2231-2258.

30. Żur KK. Quasi-Green's function approach to free vibration analysis of elastically supported functionally graded circular plates. Composite Structures 2018;183:600-610.

31. Abrate S. Functionally graded plates behave like homogenous plates. Composites Part B 2008;39:151-158.

32. Reddy JN, Wang CM, Kitipornchai S. Axisymmetric bending of functionally graded circular and annular plates. European Journal of Mechanics A/Solids 1999;18:185-199.

33. Wattanasakulong N, Chaikittiratana A. Flexural vibration of imperfect functionally graded beams based on Timoshenko beam theory: Chebyshev collocation method. Meccanica 2015;50:1331-1342.

34. Delale F, Erdogan F. The crack problem for a non-homogeneous plane. ASME Journal of Applied Mechanics 1983;50:609-614.

35. Reddy JN. Theory and analysis of elastic plates and shells. CRC Press 2006.

36. Wu TY, Liu GR. Free vibration analysis of circular plates using generalized differential quadrature rule. Computer Methods in Applied Mechanics and Engineering 2002;191:5365-5380.

37. Yalcin HS, Arikoglu A, Ozkol I. Free vibration analysis of circular plates by differential transformation method. Applied Mathematics and Computation 2009;212:377-386.

38. Zhou ZH, Wong KW, Xu XS, Leung AYT. Natural vibration of circular and annular thin plates by Hamiltonian approach. Journal of Sound and Vibration 2011;330:1005-1017.

39. Duan G, Wang X, Jin Ch. Free vibration analysis of circular thin plates with stepped thickness by the DSC element method. Thin-Walled Structures 2014;85:25-33. 